\title{
Article
}

\section{HIV propagation in a population group considering infection in the immune system of each infected individual}

\author{
Sol de Amor Vásquez-Quintero ${ }^{1}$ (D), Hernán Darío Toro-Zapata ${ }^{2 *(1)}$ and Dennis \\ Alexander Prieto-Medellín 3 (i) \\ 1 Licenciatura en Matemáticas, Universidad del Quindío; svasquezq@uqvirtual.edu.co \\ 2 Licenciatura en Matemáticas, Universidad del Quindío; hdtoro@uniquindio.edu.co \\ 3 Gerontología, Universidad del Quindío; daprieto@uniquindio.edu.co \\ * Correspondence: hdtoro@uniquindio.edu.co; Tel.:+57-3013987980
}

\begin{abstract}
A multiscale mathematical model is proposed seeking to study the propagation dynamics of the Human Immunodeficiency Virus (HIV) in a group of young people between 15 and 24 years of age, through sexual contact without protection, considering the use of antiretroviral therapy (ART) and therapeutic failure. The model consists in a scale-free complex network that follows a power law, coupled with the immunological dynamics of each individual, that is, it considers the infection by the virus in the immune system of each HIV carrier, through a system of non-linear differential equations that govern the infection's behavior in the immune system. Propagation of the virus in the network is modelled by taking into account information from the immunological status of each person. The study found that for a population to have high HIV prevalence, it is not necessary at the beginning of the simulation time for the virus to propagate rapidly. In addition, the study proves that with a higher number of sexual partners, there will be greater prevalence of HIV in the population and that the use of ART helps to control the propagation of the infection in the population. As an interesting result, it was also found that there is a higher number of HIV carriers who abandon ART than those who have access to it.
\end{abstract}

Keywords: Multi-scale model; system of differential equations; HIV propagation; complex network; basic reproduction number; antiretroviral therapy; prevalence.

\section{Introduction}

The Human Immunodeficiency Virus (HIV) has managed to propagate and remain in the human population for decades; it is one of the most serious public health problems globally, has no cure, and each year takes new lives when the infection goes on to become an acquired immunodeficiency syndrome (AIDS) [1,2]. Additionally, as time passes, not only does increase the number of infected people, but also every year increases the number of new infections [3].

The HIV is a virus that attacks the immune system of the infected person, specifically, it attacks and destroys the CD4 T lymphocytes, which are a type of cell in charge of the fabrication of antibodies to combat infections caused by external agents [4]. Once the virus enters the person's organism, it is recognized by the body's defense systems and starts the infection process. First, it adheres to healthy CD4 $\mathrm{T}$ cell, then, the virus introduces its genetic material and viral components in the cell nucleus that then begins replication (after immunological activation) of the virus within it, goes into the blood stream, and starts propagating throughout the body until infecting other healthy CD4 T cells [5].

One of the populations most vulnerable to acquire the virus are young people between 15 and 24 years of age; different studies reveal that it is in the stage when they start an active sex life, may have a greater number of sex partners, engage in sexual unprotected intercourse, and incur in the use of drugs and alcohol that are scenarios of increased risk; furthermore, many young people ignore how HIV is transmitted and most fear being tested to know their serological status [6-8]. This is how one third 
of new infections occur in this age range globally [9] and in Colombia, for 2019, $24.8 \%$ of those infected were young people [10]. This is why it is fundamental to conduct research that contributes to the study of the epidemic, taking this age group as object of study.

ART consists in the combination of two or more medications that help to maintain low levels of viral load, stable levels of healthy CD4 T cells, and diminish the probability of infecting other people [11]. For 2019, of the 38 million people who lived with HIV in the entire world, 25.4 million had access to ART, that is, $66 \%$ of those infected [3]. Unfortunately, not everyone manages to have access to ART because they do not know their serological status; more so, many factors cause people, in spite of using ART, to abandon it, among the known factors, there are the high costs of medications, side effects, and non-adherence to therapy [12]. Due to the aforementioned, in a population model for the propagation and control of HIV, it is convenient to consider access and adherence to ART.

Prior research has sought to understand the behavior of the virus in the immunological and/or population scale. This is the case of [13] that studies the dynamics of infection by HIV through stochastic birth and death processes, and from the systems of differential equations that represent a real system. Additionally, [14] shows the behavior of the virus in a population of susceptible people, people infected without ART, and people infected with ART, they propose a mathematical model based on ordinary differential equations that interprets the effect that effective access and therapeutic failure have on said populations. Finally, a study that elaborates a mathematical model for the dynamics of the HIV transmission centered on the functions of the immune response cells and considering active and inactive cytotoxic immune cells was conducted by [15], which evaluates two different scenarios of the infection (early stage and advanced stage) and employs the optimal control theory.

During the last two decades, there has been an increase in the study and use of complex networks in different contexts [16]; particularly, complex networks have gained strength when representing sexual contacts among people. We, herein, introduce a model that uses complex networks to study the dynamics of HIV propagation in people between 15 and 24 years of age, who interact through sexual contacts, considering at the same time the dynamics of infection in the immune system of each young person; the immune system is modelled through the system of ordinary differential equations (ODE) proposed in [15]. The research gains importance by being a multiscale model that integrates two components: the populational (network) and the immunological (ODEs' system), because the populational dynamics cannot be detached from the immunological.

The study permits obtaining some standard results that essentially validate the model, for example, that the infection is controlled when reducing the values of the probability of healthy CD4 T cells becoming infected and increasing the probability of active cytotoxic cells of eliminate infected CD4 $\mathrm{T}$ cells. In addition, when using reverse transcriptase inhibitors (RTI) and protease inhibitors (PI), the rate of virus propagation is reduced and the infection is also controlled in the immune system, obtaining better results when these medications are used in combination. Moreover, it is proven that with a greater number of sex partners a person has within a specific population, there will be greater prevalence of the virus within said population and the infection will propagate at higher rates, as long as the sexual encounters take place without protection. A result not at all trivial, is that to have a population with high prevalence of HIV carriers, it is not necessary for the virus to propagate rapidly at the start of the propagation process. It is observed in all the network simulations performed that the number of HIV carriers without ART was higher than the number of HIV carriers with ART, which indicates a higher number of HIV carriers who abandon ART than those who have access to it.

This paper is carried out in the following manner: section 2 describes the population model (the sexual contact network) and presents the immunological model. Section 3 conducts the respective local stability analysis of the immunological model, discussing the invariance region, equilibrium points, and the basic reproduction number. The local stability is illustrated through simulations performed for the immunological model, varying the different parameters; then, the coupling algorithm of the immunological scale with the populational is formulated. Finally, simulations are performed of the 


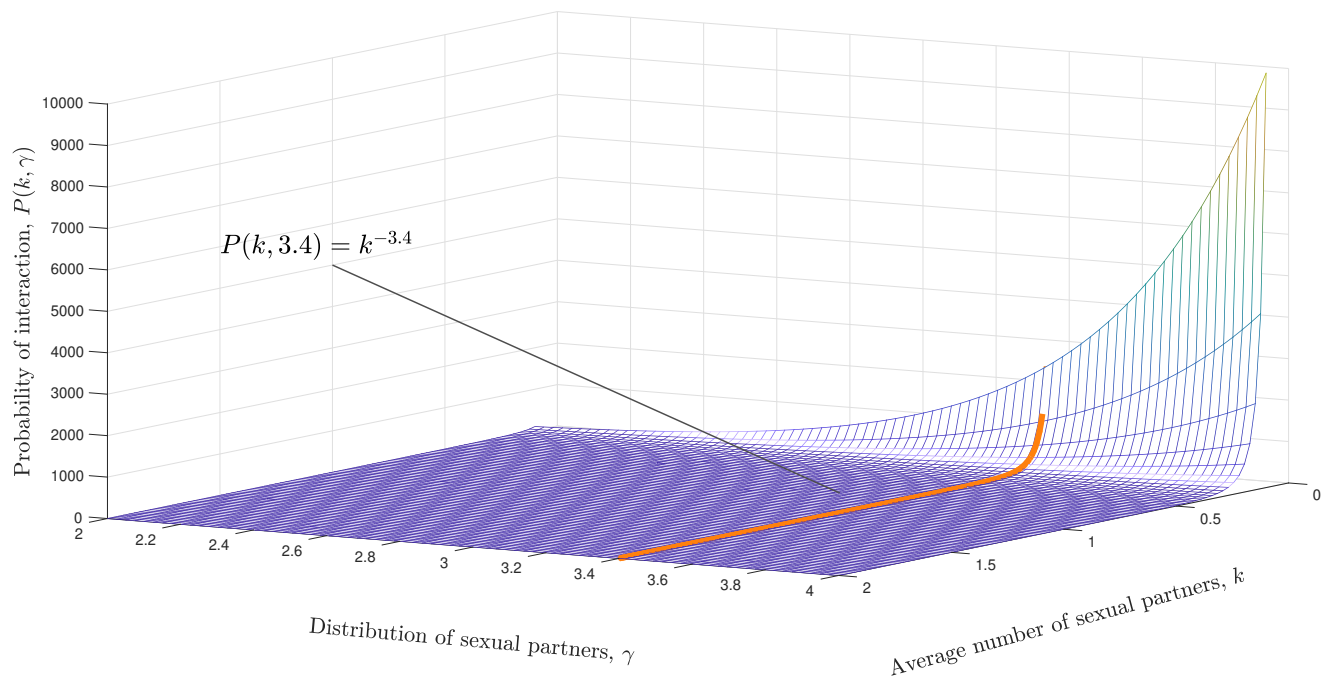

Figura 1. Graphic of $P$ in space $(k, \gamma, P)$, to $k \in[a, b]$ and $\gamma \in[a, b]$. The orange curve represents the fixed value $\gamma=3.4$ used in the simulations in this study.

coupled model, that is, the complex network. Section 4 exposes the conclusions obtained and in the end the references used are shown.

\section{Materials and Methods}

\subsection{Population model: sexual contact network}

The population of study are young people between 15 and 24 years of age and is modelled through a complex network, where the vertices represent people and the edges are the potential unprotected sexual encounters with each other, through which the virus can propagate. No differentiation is made regarding gender, sexual orientation; nor does the study consider the use of stimulants, like hallucinogenic drugs or alcohol. The model considers a constant and finite population $N$, hence, it does not take into account new young people entering the network, nor their deaths. A cluster scale-free network is generated (according to the Barabási-Albert model, being scale-free means that there is no preferred scale or preferred number of edges for each vertex with respect to the rest [17]), in which the probability $P(k, \gamma)$ of a vertex in the network interacting with other vertices decays through a power law $P(k, \gamma) \sim k^{-\gamma}$ [17], where $k$ is the average number of sex partners and $\gamma$ is the partners' distribution. Besides, the network is random non-directed and uses the algorithm by Holm and Kim [18].

Figure 1 shows the plot of $P$ in the $(k, \gamma, P)$-space, where the orange curve corresponds to the fixed value $\gamma=3.4$ used in the simulations in this study. Figure 1 shows how the probability $P(k, \gamma)$ diminishes as the average number of sexual partners increases; also, at a low average number of sexual partners and high values in the distribution of said partners, the probability of a vertex of the network to interact with others will be higher.

Use of ART is taken into account, considering that for a person to have access to it, first must have to be tested, diagnosed as a carrier, and having the economic resources (these can be their own or from health insurance) to acquire the medications. The model includes non-adherence to therapy that can be caused by consumption of psychoactive substances, depression, low level of schooling, little trust in the benefits of the therapy, greater number of tablets and dosage, lack of family and/or social support, etc. [19], and also considers therapeutic failure, which has drug resistance, drug toxicity (degree of adverse effects) and non-observance of ART as principal causes [20].

The study comprised a population of $N_{j}$ young people between 15 and 24 years of age, where $x_{i}=x_{i}(\tau), y_{i}=y_{i}(\tau)$ and $z_{i}=z_{i}(\tau)$ are the number of young people susceptible of acquiring the virus, 


\begin{tabular}{||c|l|l||}
\hline Sím. & Description & Values used \\
\hline \hline$k$ & Average number of sex partners & {$[1-10]$} \\
$\gamma$ & Distribution of sex partners & 3.4 \\
$N_{j}$ & Number of susceptible young people, carriers with ART and carriers without & 1000 \\
& ART & {$[1-200]$} \\
$\tau$ & Discrete simulation time in weeks & - \\
$t_{f}$ & Number of iterations on the network & - \\
$x_{i}$ & Number of young people susceptible of acquiring the virus & - \\
$y_{i}$ & Number of young people carriers of the virus with ART & - \\
$z_{i}$ & Number of young people carriers of the virus without ART & \\
\hline
\end{tabular}

Cuadro 1. Description of the parameters of the population model that will be used in the simulation. The intervals were assumed randomly and obtained from [16,22].

young carriers of the virus who use ART and young carriers who do not use ART, respectively, where $\tau$ is the discrete simulation time and we define $t_{f}$ as the final simulation time, thus, $\tau=0, \ldots, t_{f}$. Finally, $x_{i}=1, \ldots, m, y_{i}=1, \ldots, n$ and $z_{i}=1, \ldots, l$, then the relation $m+n+l=N_{j}$ holds for every $\tau$ since the population is assumed constant over time, an oversimplification that can be relaxed in future a work.

The complex network is defined by the $\{G, \Gamma\}$ pair, where $G$ is a graph, that is, an ordered pair of disjointed sets $\langle V, A\rangle$ that correspond to the set of vertices $V$ and edges $A$, and $\Gamma$ an evolution operator that determines changes of the network [21]; that is,

$$
\left\{\begin{array}{l}
\langle V, A\rangle_{\tau+1}=\Gamma\left(\langle V, A\rangle_{\tau}\right) \\
\langle V, A\rangle_{0}=\left\langle V_{0}, A_{0}\right\rangle, \quad \tau=0, \ldots, t_{f}
\end{array}\right.
$$

where, $\langle V, A\rangle_{0}=\left\langle V_{0}, A_{0}\right\rangle$ is the initial configuration of vertices and edges of the network at $\tau=0$. Additionally, the configuration $\langle V, A\rangle$ over time $\tau+1$ is given by the $\Gamma$ operator applied in the previous iteration $\tau$, with which is achieved a "sequence" of networks that correspond to iterations over time. The evolution operator $\Gamma$ governing the network changes implicitly considers two fundamental aspects:

1. Infection by the virus in the immunological scale.

2. Defines the virus propagation in the populational scale, considering aspects from the immunological scale.

The coupling of both dynamics takes place aiming to achieve a somewhat more realistic model where the events in the populational scale are affected by the state of the infection in the immune system of each infected individual in the population. The parameters and variables considered to define changes in the network are the effective use and abandonment of ART, the average viral load and number of healthy CD4 T cells of each infected person (details of the coupling algorithm are described in the Results section). Table 1 shows the parameters used in the populational model with their respective values used in simulations.

\subsection{Model for HIV infection in the immunological scale}

A mathematical model that considers the dynamics of the HIV infection while using ART has been described in [15], which will be used in this work. The following describes the model and the Appendix contains the local stability analysis. Use of ART seeks to reduce the number of new infected cells through RTI and PI, which are denoted, respectively, by $u_{1}$ and $u_{2}$, such that $u_{i} \in[0,1]$ for $i=1,2$. When $u_{i}=1$, it means that the use of the medication was efficient by $100 \%$ and $u_{i}=0$ represents a $0 \%$ efficiency or absence of ART.

In the first place, $T=T(t)$ is the average number of healthy CD4 T cells in a time $t$, these cells are produced at a constant rate $\sigma$ in the organism and their natural death is described by the rate $\mu$, so 
that $\mu T$ represents the average of healthy CD4 T cells that die naturally. CD4 T cells are infected when coming in contact with the viral particles $V=V(t)$ over time, at a probability $\beta$. The mass action principle permits establishing that the average of new infected cells is proportional to the product of healthy CD4 T cells with the infectious viral particles [13]; this permits assuming that the average number of cells that become infected is $\beta\left(1-u_{1}\right) T V$, where $\left(1-u_{1}\right)$ is the failure rate of the RTI. The equation to describe the variation of healthy CD4 $\mathrm{T}$ cells with respect to time $t$ is:

$$
\dot{T}=\sigma-\beta\left(1-u_{1}\right) T V-\mu T
$$

The average number of infected cells over time is denoted $T^{*}=T^{*}(t)$; which die at a rate $\delta$; thus, $\delta T^{*}$ is the average number of infected cells that die due to causes associated with the virus. This death is known as cellular lysis, consisting in the rupture of the cell membrane. The differential equation that describes the variation of infected CD4 T cells is:

$$
\dot{T}^{*}=\beta\left(1-u_{1}\right) T V-\gamma T^{*} M^{*}-\delta T^{*}
$$

To explain the term $\gamma T^{*} M^{*}$ consider that $T^{*}$ cells stimulate the specific cell immune response against HIV (CD8 T cells) that are inactive $M=M(t)$ and become active $M^{*}=M^{*}(t)$. The CD8 $\mathrm{T}$ cells recognize and destroy only the infected cells through direct attack, injecting toxic enzymes that cause their destruction [23,24]. Thus, $M$ is the average number of CD8 T immune cells que are inactive in a time $t$ and $M^{*}$ represents the average number of activated CD8 T immune cells in a time $t$. The $M^{*}$ cells are in charge of destroying the $T^{*}$ cells with a probability $\gamma$, then $\gamma T^{*} M^{*}$, through the mass-action principle, is the average number of infected cells destroyed by the specific cell immune response. The CD8 T cells are produced in the organism at a constant rate $\lambda$ and the average of cells dying naturally is described by $\rho M$, where $\rho$ is the death rate of the CD $8 \mathrm{~T}$ cells. It is assumed that the influence of the $T^{*}$ cells stimulates the active immune response $M^{*}$ according to the law of mass action at a probability $\psi$, hence, the average number of inactive $M$ cells that activate against HIV is given by $\psi T^{*} M$. Thus, the equation for the average number of $M$ cells remains:

$$
\dot{M}=\lambda-\psi T^{*} M-\rho M
$$

Furthermore, it is considered that proliferation of activated CD8 T cells at a probability $\alpha$ stimulated by the presence of infected cells $T^{*}$ then, through the mass-action principle, $\alpha T^{*} M^{*}$ represents the average of new active CD8 T cells produced through proliferation. Finally, it is kept in mind that cells from the active cell response $M^{*}$ die at a rate $\rho$, thus, $\rho M^{*}$ is the average of $M^{*}$ cells that die naturally. Due to the aforementioned, the differential equation is:

$$
\dot{M}^{*}=\alpha T^{*} M^{*}+\psi T^{*} M-\rho M^{*}
$$

When the $T^{*}$ cells die due to cellular lysis, their cell membranes rupture, produce the liberation of intracellular material and release a large amount of new infectious and non-infectious virions (the latter as long as the PI are used); we denote $V=V(t)$ the average number of infectious viral particles in a time $t$ and $W=W(t)$ the average number of non-infectious viral particles in a time $t$. The elimination rate of viral particles is represented with $c$; hence, $c V$ and $c W$ represent the average number of infectious and non-infectious viral particles eliminated, respectively. In the model, replication of $V$ and $W$ is described through $N \delta T^{*}$ in which $N$ is the average number of viral particles produced by an infected $T^{*}$ cell and the production of non-infectious virions depends on the use of PI (with effectiveness $\left.u_{2}\right)$. Therefore, expressions $N \delta\left(1-u_{2}\right) T^{*}$ and $N \delta u_{2} T^{*}$ represent the average number of infectious and non-infectious virions, respectively, produced by each infected cell, bearing in mind the effect of ART. The differential equations that describe this behavior are:

$$
\dot{V}=N \delta\left(1-u_{2}\right) T^{*}-c V
$$




\begin{tabular}{||c|l|l|c||}
\hline Sím. & Description & Values used & Ref. \\
\hline \hline$\sigma$ & Production rate of healthy CD4 T cells & $10 \mathrm{~mm}^{3} \mathrm{~d}^{-1}$ & {$[25,26]$} \\
$\beta$ & CD4 T cells infection probability & $2.5 \times 10^{-5} \mathrm{~mm}^{3} \mathrm{~d}^{-1}$ & {$[26]$} \\
$\mu$ & Death rate of the uninfected CD4 T cells & $1 \times 10^{-2} \mathrm{~d}^{-1}$ & {$[25]$} \\
$\delta$ & Death rate of infected CD4 T cells & $0.26 \mathrm{~d}^{-1}$ & {$[25,26]$} \\
$N$ & Production rate of viral particles & $500 \mathrm{~d}^{-1}$ & {$[25]$} \\
$c$ & Rate of virus elimination & $2.4 \mathrm{~d}^{-1}$ & {$[26]$} \\
$\psi$ & Activation probability of CD8 T cells & $2 \times 10^{-3} \mathrm{~mm}^{3} \mathrm{~d}^{-1}$ & - \\
$\alpha$ & Replication probability of the active CD8 T cells & $5 \times 10^{-5} \mathrm{~mm}^{3} \mathrm{~d}^{-1}$ & {$[26]$} \\
$\rho$ & Death rate of CD8 T cells & $0.1 \mathrm{~mm}^{3} \mathrm{~d}^{-1}$ & {$[25,26]$} \\
$\gamma$ & Probability of the active CD8 T cells eliminating the infected CD4 T & $2 \times 10^{-3} \mathrm{~mm}^{3} \mathrm{~d}^{-1}$ & {$[26]$} \\
$\lambda$ & cells & $5 \mathrm{~mm}^{3} \mathrm{~d}^{-1}$ & {$[26]$} \\
$u_{1}$ & Production rate of inactive CD8 T cells & - & - \\
$u_{2}$ & Effectiveness of RTI & - & - \\
$T_{0}$ & Initial value of the healthy CD4 T cells & $1000 \mathrm{~mm}^{3}$ & {$[26]$} \\
$T_{0}^{*}$ & Initial value of infected CD4 T cells & $0 \mathrm{~mm}^{3}$ & {$[26]$} \\
$M_{0}$ & Initial value of inactive CD8 T cells & $1 \mathrm{~mm}^{3}$ & {$[26]$} \\
$M_{0}^{*}$ & Initial value of active CD8 T cells & $1 \times 10^{3} \mathrm{~mm}^{3}$ & {$[26]$} \\
$V_{0}$ & Initial infectious viral load & {$[26]$} \\
$W_{0}$ & Initial non-infectious viral load & - & - \\
\hline
\end{tabular}

Cuadro 2. Description of parameters for model (7) and baseline values used in the simulation, with their respective source.

and

$$
\dot{W}=N \delta u_{2} T^{*}-c W
$$

With the assumptions described, the system that models HIV replication in an infected person's organism considering ART is given by the system (7) of differential equations. Table 2 describes the parameters used in the immunological model.

$$
\left\{\begin{array}{l}
\dot{T}=\sigma-\beta\left(1-u_{1}\right) T V-\mu T \\
\dot{T}^{*}=\beta\left(1-u_{1}\right) T V-\gamma T^{*} M^{*}-\delta T^{*} \\
\dot{M}=\lambda-\psi T^{*} M-\rho M \\
\dot{M}^{*}=\alpha T^{*} M^{*}+\psi T^{*} M-\rho M^{*} \\
\dot{V}=N \delta\left(1-u_{2}\right) T^{*}-c V \\
\dot{W}=N \delta u_{2} T^{*}-c W
\end{array}\right.
$$

The model (7) was proposed in [15] but its local stability was not analyzed with control; here, we perform this analysis and use it to describe the immune system of each person in the complex network.

\section{Results}

\subsection{Analysis of the local stability of the immunological model}

The model (7) is defined in an invariance region whose importance is to guarantee that the populations do not become negative or grow indefinitely, in other words, it guarantees that the model's solutions make biological sense. Mathematically, the region is a compact set that finally $(t \geq 0)$ will contain the system's solutions, that is, ensures that the system's solutions (7) enter the region and remain in it for all $t \geq 0$. The demonstration of the Proposition 1 is found in the Appendix. 
Proposition 1. If $\gamma \geq \alpha$, the system (7) is defined in the positively invariant region $\Omega=$ $\left\{x \in \mathbb{R}^{6} \mid 0 \leq T \leq \frac{\sigma}{\mu}, 0 \leq G \leq \frac{\sigma+\lambda}{\varepsilon}, 0 \leq D \leq \frac{N \delta(\sigma+\lambda)}{\varepsilon c}\right\}$, where $x=\left(T, T^{*}, M, M^{*}, V, W\right) ; G=T+$ $T^{*}+M+M^{*}$ and $D=V+W$.

Condition $\gamma \geq \alpha$ refers to the probability of the probability of the active CD8 T cells eliminating the infected CD4 T cells being higher than the probability of replicating the active CD8 T cells.

The stationary solutions or equilibrium points represent the values of variables $T, T^{*}, M, M^{*}$, $V$, and $W$ where there is no change in the system's behavior; that is, they are constant solutions $\bar{E}=\left(\bar{T}, \bar{T}^{*}, \bar{M}, \bar{M}^{*}, \bar{V}, \bar{W}\right)$ of system $(7)$ whose coordinates are:

$$
\begin{array}{ll}
\bar{T}=\frac{\sigma c}{N \delta \beta\left(1-u_{1}\right)\left(1-u_{2}\right) \bar{T}^{*}+c \mu}, & \bar{M}=\frac{\lambda}{\psi \bar{T}^{*}+p}, \\
\bar{M}^{*}=\frac{\lambda \psi \bar{T}^{*}}{\left(\psi \bar{T}^{*}+\rho\right)\left(\rho-\alpha \bar{T}^{*}\right)}, & \bar{V}=\frac{N \delta\left(1-u_{2}\right) \bar{T}^{*}}{c}, \\
\bar{W}=\frac{N \delta u_{2} \bar{T}^{*}}{c}, &
\end{array}
$$

From (8) for $\bar{T}^{*}=0$, the virus free equilibrium $\bar{E}_{0}=\left(\frac{\sigma}{\mu}, 0, \frac{\lambda}{\rho}, 0,0,0\right)$ is obtained, where $\frac{\sigma}{\mu}$ represents the average number of healthy cells $T$ and $\frac{\lambda}{\rho}$ represents the average number of inactive CD8 T cells. On the other hand, to have equilibria in precense of virus, note that it is required $\rho>\alpha \bar{T}^{*}$ to have a non-negative $\bar{M}^{*}$. In (8) we denote $\bar{T}^{*}$ the solution of the cubic equation:

$$
A \xi^{3}+B \xi^{2}+C \xi+D=0
$$

where,

$$
\begin{array}{ll}
A=\delta \alpha \psi F, & B=-\gamma \lambda \psi F+\delta F \rho(\alpha-\psi)+\delta \alpha \psi c \mu-\alpha \psi \sigma F, \\
C=-\delta \rho^{2} F-c \mu \gamma \lambda \psi+\delta \rho c \mu(\alpha-\psi)+F \rho \sigma(\psi-\alpha), & D=-\delta \rho^{2} c \mu+\sigma \rho^{2} F,
\end{array}
$$

and $F=N \delta \beta\left(1-u_{1}\right)\left(1-u_{2}\right)$. To study the system's behavior in presence of viremia through a stability analysis of system (7), it is convenient to find the basic reproduction number $R_{0}$, which corresponds to the average number of secondary cells infected in a susceptible population by an infected cell with certain initial viral load [13]. Mathematically, $R_{0}$ is the spectral radius of the next generation matrix evaluated in the virus free equilibrium [27] (See Appendix). For system (7), it is given explicitly by:

$$
R_{0}=\frac{N \beta\left(1-u_{1}\right)\left(1-u_{2}\right)}{c} \bar{T}_{0}
$$

The $R_{0}$ is important given that "theoretically" it determines if there will be a peak in viral production $\left(R_{0}>1\right)$, on the contrary, there will be no increase of the viral particles when $R_{0}<1$, which means that the infection is not established and manages to disappear, as established in the following results $[28-30]$.

Proposition 2. If $R_{0}>1$, there is a single equilibrium in presence of virus given by $\bar{E}_{1}=$ $\left(\bar{T}, \bar{T}^{*}, \bar{M}, \bar{M}^{*}, \bar{V}, \bar{W}\right)$.

The demonstration of the Proposition 2 is found in the Appendix.

The analysis of local stability of the virus free equilibrium $\bar{E}_{0}$ permits determining conditions for the system's solutions to stabilize close to such values or approach them over time, i.e., determine "theoretical" conditions under which the infection do not manages to establish in the immune system: 

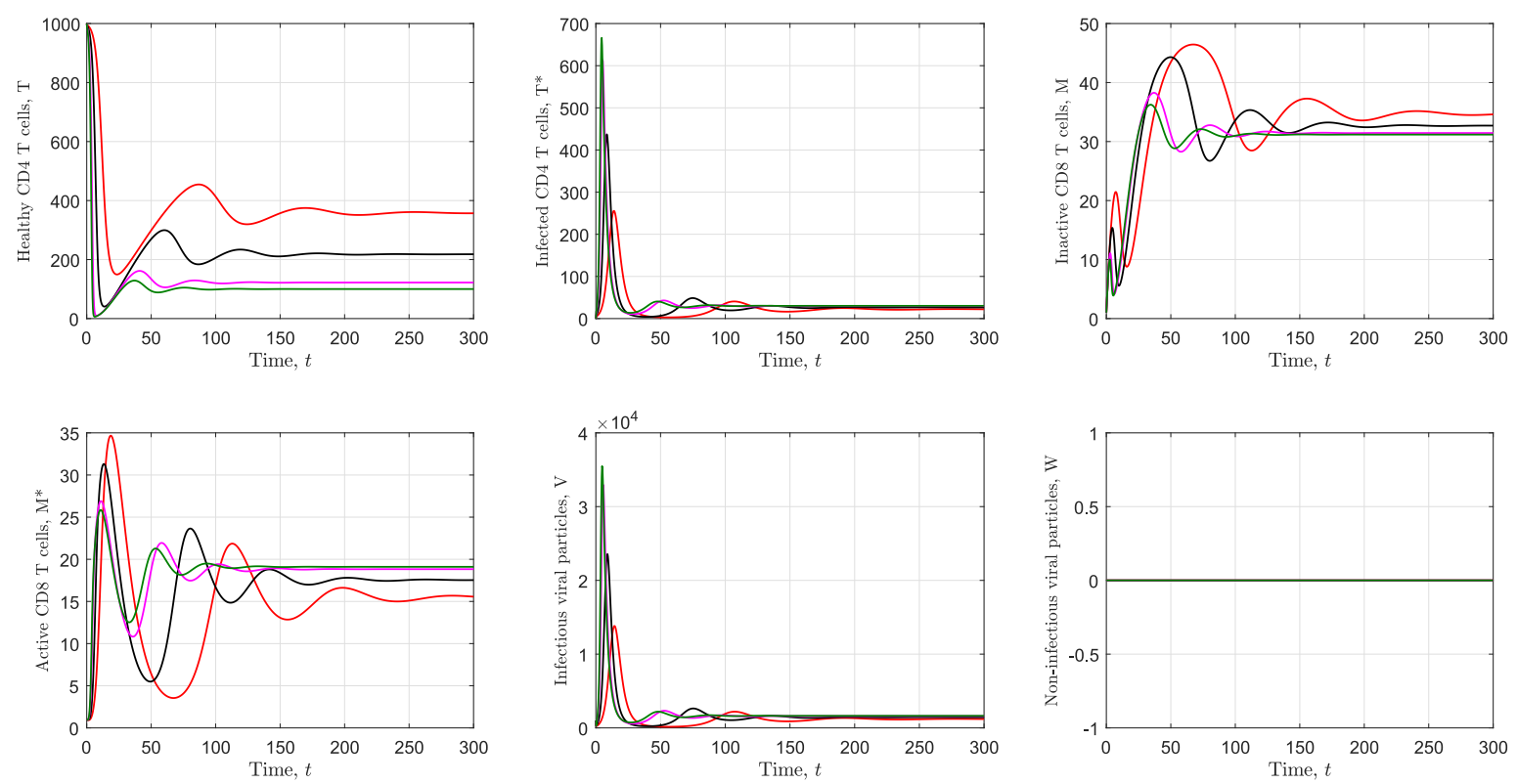

Figura 2. Numerical solutions of system (7), considering the parameters of the Table 2, simulating 300 days, varying the the CD4 T cells infection probability, $\beta$, taking as fixed the value of the probability of the active CD8 T cells eliminating the infected CD4 T cells, $\gamma=2 \times 10^{-3}$ and considering that ART is not used, that is, the RTI and PI are in null values, $u_{i}=0$ with $i=1,2$. When $\beta=1.5 \times 10^{-5}$ is the red curve with $R_{0}=1.2168, \beta=2.5 \times 10^{-5}$ is the black curve with $R_{0}=2.0280, \beta=4.5 \times 10^{-5}$ is the magenta curve with $R_{0}=3.6504$, and $\beta=5.5 \times 10^{-5}$ is the green curve with $R_{0}=4.4616$.

Proposition 3. The virus free equilibrium $\bar{E}_{0}=\left(\frac{\sigma}{\mu}, 0, \frac{\lambda}{\rho}, 0,0,0\right)$ is locally asymptotically stable if and only if $R_{0}<1$.

This means that if a person is susceptible of acquiring HIV, but satisfies $R_{0}<1$, then the infection "theoretically" is not established in the immune system, and the initial viral load becomes undetectable; this result is theoretical given that infection by HIV has no cure.

Because it was not possible to perform the demonstration analytically due to the algebraic difficulty, it is conjectured that equilibrium $\bar{E}_{1}=\left(\bar{T}, \bar{T}^{*}, \bar{M}, \bar{M}^{*}, \bar{V}, \bar{W}\right)$ is locally asymptotically stable when $R_{0}>1$. The Appendix presents the demonstration of Proposition 3 and the conjecture will be illustrated numerically via simulation.

\subsection{Simulation of the immunological model}

For the purpose of seeing the impact of some parameters on the system, and analyzing which of them can be intervened for the infection level in the immune system to be low or null, the numerical simulation of system (7) is performed by using the parameter values in Table 2 and varying the CD4 T cells infection probability $\beta$, and the probability of the probability of the active CD8 T cells eliminating the infected CD4 T cells $\gamma$ (Figures 2 and 3).

Figure 2 shows the variation of the CD4 T cells infection probability, $\beta$. A fixed value was used for $\gamma=2 \times 10^{-3}$, considering the values $u_{1}=0$ and $u_{2}=0$ that represent the effectiveness of RTI and PI, respectively, which implies that there is no use of medications to control the infection. The red curve corresponds to $\beta=1.5 \times 10^{-5}$, the black curve to $\beta=2.5 \times 10^{-5}$, finally, the magenta and green curves represent $\beta=4.5 \times 10^{-5}$ and $\beta=5.5 \times 10^{-5}$, respectively. It may be noted in the red curve (for the smaller value $\beta=1.5 \times 10^{-5}$ ) that the number of $T$ cells is kept at higher levels than $150 \mathrm{cell} / \mathrm{mm}^{3}$ and stabilize at 358 cells. The green curve shows that for the highest value of $\beta=5.5 \times 10^{-5}$, the $T$ cells remain at levels below $200 \mathrm{cell} / \mathrm{mm}^{3}$ and stabilize at $100 \mathrm{cell} / \mathrm{mm}^{3}$. With respect to the number of infected cells $T^{*}$, it was observed that these stabilized in the interval of $(1,40) \mathrm{cell} / \mathrm{mm}^{3}$, for all the 

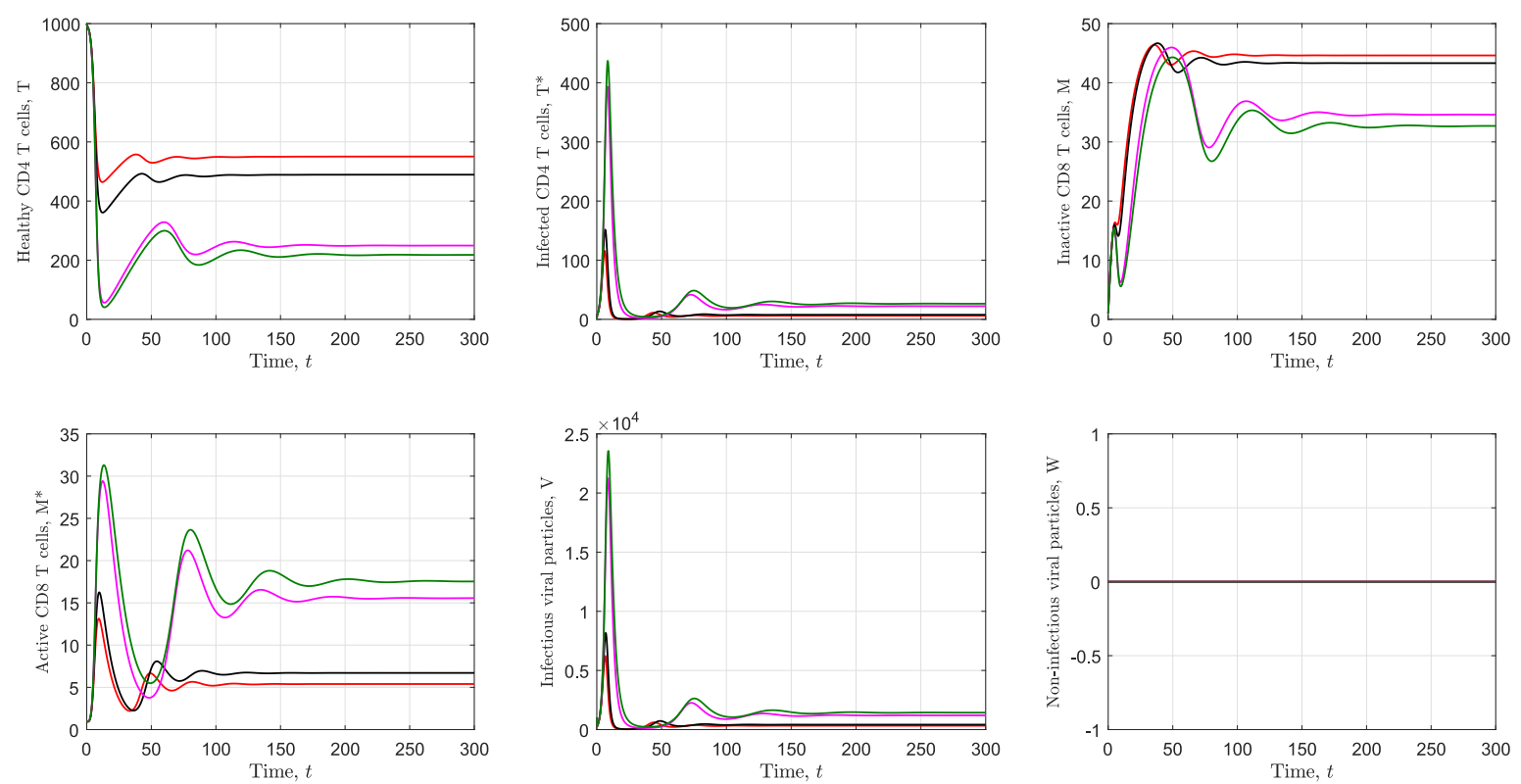

Figura 3. Simulations performed for the parameters according to the values from Table 2, varying the probability of the active CD8 $\mathrm{T}$ cells eliminating the infected CD4 T cells $\gamma$, where $\gamma=9 \times 10^{-2}$ (red), $\gamma=6 \times 10^{-2}$ (black), $\gamma=5 \times 10^{-3}$ (magenta) and $\gamma=2 \times 10^{-3}$ (green). Fixed values were taken for the the CD4 T cells infection probability, $\beta=2.5 \times 10^{-5}$, and ART use was not considered, thus, $u_{i}=0$, for $i=1,2$. $R_{0}=2.0280$ in all the scenarios illustrated.

simulated values of $\beta$. The legend for Figure 2 shows the $R_{0}$ values obtained in each case, all greater than 1 , and it was observed that the organism remains with presence of virus during the entire simulation time (active CD8 T cells $M^{*}$, infected cells $T^{*}$, and infectious viral particles $V$ take values above zero), which illustrate the aforementioned conjeture, that is, that the equilibrium $\bar{E}_{1}=\left(\bar{T}, \bar{T}^{*}, \bar{M}, \bar{M} \bar{M}^{*}, \bar{V}, \bar{W}\right)$ is locally asymptotically stable when $R_{0}>1$. Regarding the non-infectious viral particles, it can be noted that these remain at zero during the entire simulation time, because the PI are not used $\left(u_{2}=0\right)$, which leads to no production of non-infectious viral particles.

Figure 3 shows the dynamics when varying the parameter $\gamma$, which denotes the probability of the active CD8 T cells $M^{*}$ eliminating the infected CD4 T cells $T^{*}$. The simulation considers a fixed value for the infection probability $\beta=2.5 \times 10^{-5}$ and null values for the RTI and PI effectiveness $\left(u_{i}=0\right.$, for $i=1,2$ ), that is, ART is not used. When taking large values of $\gamma$, i.e., $\gamma=9 \times 10^{-2}$ (red curve) the $T$ cells tend to stabilize at 525 cell $/ \mathrm{mm}^{3}$ approximately, infected cells $T^{*}$ stabilize at $15 \mathrm{cell} / \mathrm{mm}^{3}$ and infectious viral particles $V$ do so at $1000 \mathrm{vir} / \mathrm{mm}^{3}$, where they remain for most of the simulation time. On the contrary, for lower values of $\gamma$, the scenario was quite different, as expected, due to the diminished probability of the infected cells $T^{*}$ of being annihilated through the action of the active CD8 $\mathrm{T}$ cells. Thus, when simulating for $\gamma=2 \times 10^{-3}$ (green curve) the healthy cells $T$ tend to stabilize at 217 cell $/ \mathrm{mm}^{3}$; infected cells $T^{*}$ do so at 30 cell $/ \mathrm{mm}^{3}$ and infectious viral particles $V$ at $3000 \mathrm{vir} / \mathrm{mm}^{3}$. It should be clarified that for all probability variations of $\gamma, R_{0}=2.0280$ was obtained, since $R_{0}$ does not depend on $\gamma$. These results also illustrates that when $R_{0}>1$, there is virus presence during all the simulation time. Note that there was no production of non-infectious viral particles, given that the PI are not used.

Observe that the variations of the infection probability of the healthy cells $T$, $\beta$, and the probability of the active CD8 T cells eliminating the infected cells $T^{*}, \gamma$, produce different scenarios for the infection, so that if the levels for $\beta$ are high and those of $\gamma$ are low, the virus will spread faster in the carrier's organism, producing an outbreak of the infection and, vice versa, when $\beta$ is low and $\gamma$ is high, the virus is not established quickly in the organism. These results basically validate the immunological model, given that they agree with known facts on the infection process and the specific cell immune response to HIV. 

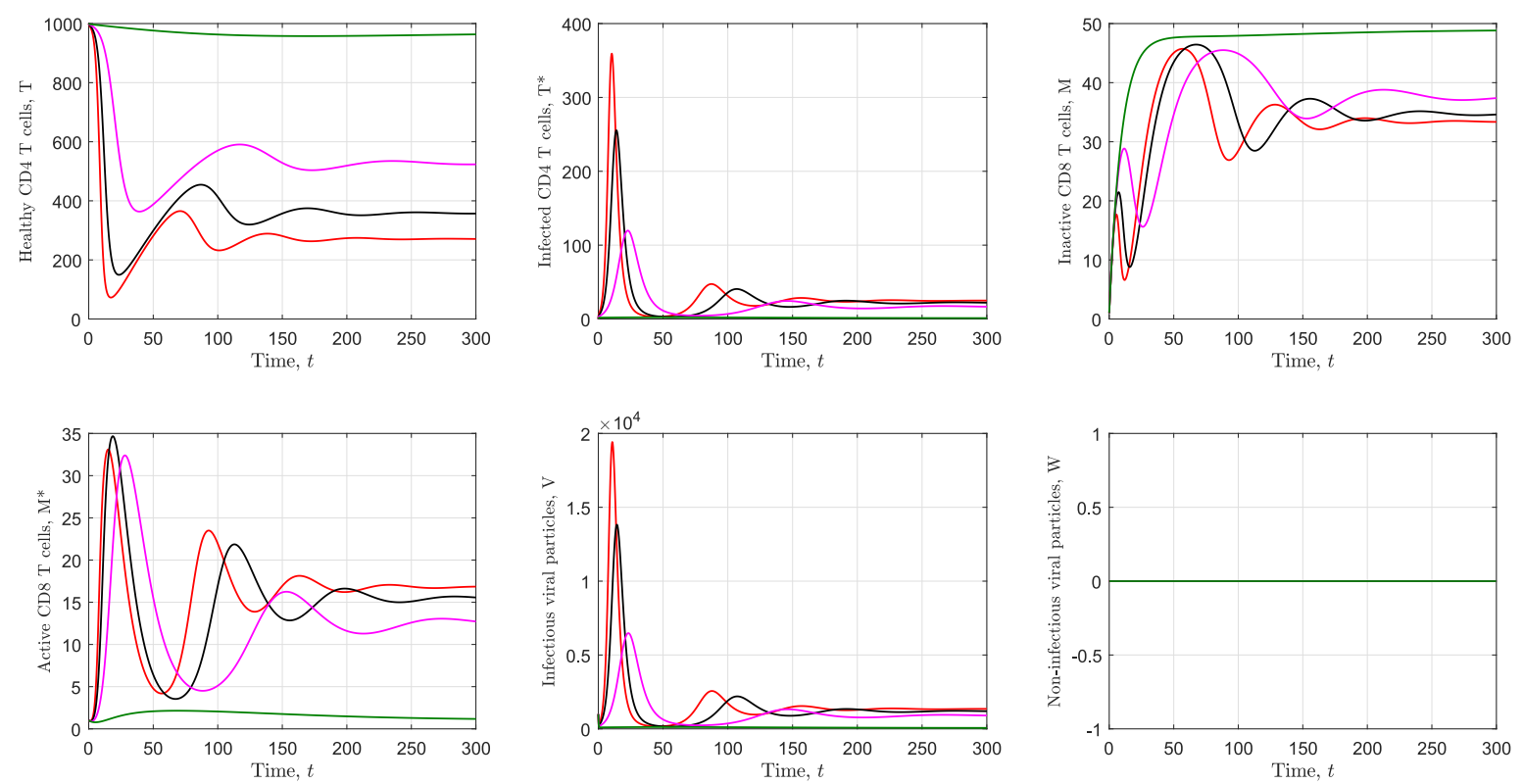

Figura 4. Dynamics of the model (7), fixed values are used for the the CD4 T cells infection probability, $\beta=2.5 \times 10^{-5}$, the probability of the probability of the active CD8 T cells eliminating the infected CD4 T cells, $\gamma=2 \times 10^{-3}$, and no use of PI is considered, $u_{2}=0$. The RTI are varied thus: $u_{1}=(0.2,0.4,0.6,0.8)$, corresponding to the colored curves (red, black, magenta, green) with their respective values of $R_{0}=$ $(1.6224,1.3168,1.0112,0.7056)$. Simulations were performed in the interval of 300 days.

Finally, simulations of system (7) are conducted varying the use of ART to control the infection (Figures 4-6), that is, the parameters that represent the effectiveness of RTI $\left(u_{1}\right)$ and PI $\left(u_{2}\right)$ are varied. Figure 4 simulates different scenarios for ART that use only the RTI, that is, varies the value of $u_{1}$ but $u_{2}=0$. The values simulated for $u_{1}$ were $0.2,0.4,0.6$ and 0.8 represented by the curves in red, black, magenta, and green, respectively. Values were taken as fixed for the infection probability of the $T$ cells $\left(\beta=2.5 \times 10^{-5}\right)$, and for the probability of the active CD8 T cells eliminating infected cells $T^{*}\left(\gamma=2 \times 10^{-3}\right)$; due to the nonuse of PI, the number of non-infectious viral particles is null in this simulation. When simulating for high RTI effectiveness, for example $u_{1}=0.8$ (green), it is found that the infection is controlled, which is reflected by the value of $R_{0}=0.7056<1$, which means that the immune system of the individual will be (theoretically) virus free. Additionally, the levels of infectious viral particles remained almost undetectable, that is, below $50 \mathrm{vir} / \mathrm{mm}^{3}$, the number of infected cells $T^{*}$ was throughout the simulation time close to zero, and the average number of active CD8 T cells was 2 cell $/ \mathrm{mm}^{3}$. Conversely, when taking lower values for the RTI effectiveness $\left(u_{1}=0.2\right)$, the infected cells $T^{*}$ stabilize at $25 \mathrm{cell} / \mathrm{mm}^{3}$, the number of infectious viral particles $V$ do so at $1500 \mathrm{per}$ vir $/ \mathrm{mm}^{3}$, the number of active CD8 T cells $M^{*}$ at $17 \mathrm{cell} / \mathrm{mm}^{3}$, and healthy cells $T$ at 271 cell $/ \mathrm{mm}^{3}$. The red curve corresponds to $R_{0}=1.6224$, the black one to $R_{0}=1.3168$, and the magenta curve has $R_{0}=1.0112$.

The simulation for the PI alone is shown in Figure 5 and very similar results are obtained, including the resulting value of $R_{0}$. Fixed values were taken for the infection probability of the $T$ cells and the probability of the active CD8 T cells eliminating infected cells $T^{*}$; thus, $\beta=2.5 \times 10^{-5}$ and $\gamma=2 \times 10^{-3}$, respectively. The simulation uses $u_{1}=0$ and $u_{2}$ equal to $0.2,0.4,0.6$, and 0.8 represented by the red, black, magenta, and green curves, respectively. It may be observed that the viral particles $V$ reach very high levels in the organism when $u_{2} \leq 0.6$, with these being greater than $3000 \mathrm{vir} / \mathrm{mm}^{3}$. Also the equilibrium values are in the interval of $(500,2000)$, which means there is an infection outbreak (in those cases simulated $R_{0}$ was greater than 1$)$. In spite of having varied the effectiveness of PI $\left(u_{2}\right)$, the results for the infected cells $T^{*}$ were also quite similar, almost equal to those obtained in the previous simulation (Figure 4), when using values of $u_{2} \leq 0.6, T^{*}$ tends to stabilize in the interval $(5,30)$ cell $/ \mathrm{mm}^{3}$. and the active CD8 T cells stabilize in the interval $(13,17)$. The most notable difference between both simulations performed for the RTI and PI effectiveness, is that for the first (Figure 4), 

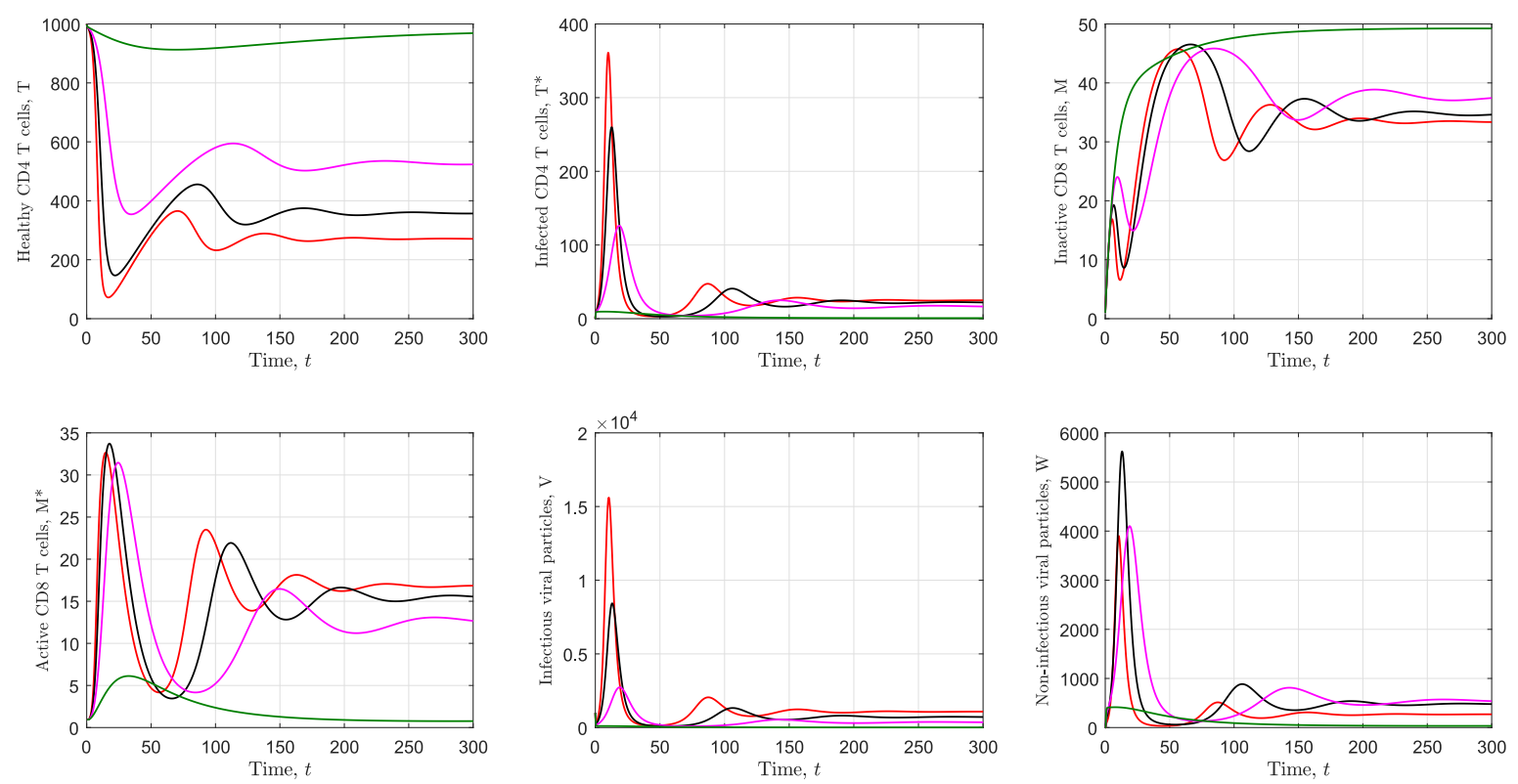

Figura 5. System simulation (7), taking the the CD4 T cells infection probability, $\beta=2.5 \times 10^{-5}$, the probability of the probability of the active CD8 T cells eliminating the infected CD4 T cells, $\gamma=2 \times 10^{-3}$ and use of RTI is not considered, $u_{1}=0$. The PI are varied, $u_{2}=(0.2,0.4,0.6,0.8)$ that correspond to the colored curves (red, black, magenta, green) with their respective values of $R_{0}=(1.6224,1.3168,1.0112,0.7056)$ in simulation time of $t=300$ days.

the levels of defective viral particles is zero for the entire time as expected (RTI act at the moment of conversion of the RNA into the viral DNA) in deed, defective viral particles are not produced, which does occur when PI are used (Figure 5). Moreover, it is observed, with the assumptions considered here, that when reaching PI effectiveness in the interval $(0.8,1)$ the viral load becomes undetectable and very high values of healthy cells $T$ are reached.

Finally, Figure 6 shows the simulation to control the virus by using RTI $\left(u_{1}\right)$ and PI $\left(u_{2}\right)$ simultaneously under the assumption that $u_{1}=u_{2}$ for convenience and simplicity. The values used were 0.2, 0.4, 0.6 and 0.8 represented by the red, black, magenta and green curves, respectively. In addition, fixed values are taken of the infection probability of the $T$ cells and the probability of the active CD 8 $\mathrm{T}$ cells eliminating the infected cells $T^{*}$, being $\beta=2.5 \times 10^{-5}$ and $\gamma=2 \times 10^{-3}$, respectively. The panorama of the infection in this simulation is totally different from that shown in Figures 4 and 5 , given that in this case HIV does not spread in the same magnitude; in deed, for values of RTI and PI effectiveness above 0.6 (magenta and green curves), the viral load is not detectable for both infectious and non-infectious viral particles, the infected cells $T^{*}$ are in values practically null and the number of active CD8 $\mathrm{T}$ cells per $\mathrm{mm}^{3}$ remains for most of the simulation time very close to zero, which is why the green and magenta curves are over the horizontal axis. Note also that healthy cells $T$ remain close to 1000 cell $/ \mathrm{mm}^{3}$ when the effectiveness in the use of RTI and PI are above 0.6. Additionally, when said effectiveness take on values lower or equal to 0.4 (red and black curves), the infected cells $T^{*}$ stabilize at interval $(1,50)$ cell $/ \mathrm{mm}^{3}$, the infectious viral particles stabilize at interval $(100,1500)$ vir $/ \mathrm{mm}^{3}$, and the non-infectious viral particles stabilize at interval $(100,500) \mathrm{vir} / \mathrm{mm}^{3}$; lastly, it is worth highlighting that the values obtained of $R_{0}$ in those cases are below 1 , as reported in the figure legend, which illustrates the result of the Proposition 3.

With the aforementioned, it can be stated that when using the different types of inhibitors to combat HIV, the results are much more encouraging if we seek to suppress viral load and diminish the rate at which the virus spreads within the organism of each carrier. With the parameters considered here, when the effectiveness of RTI and PI take on high values (over 0.6), small values for $R_{0}$ are achieved and therefore, also low levels of infected CD4 T cells, active CD8 T cells and viral particles 

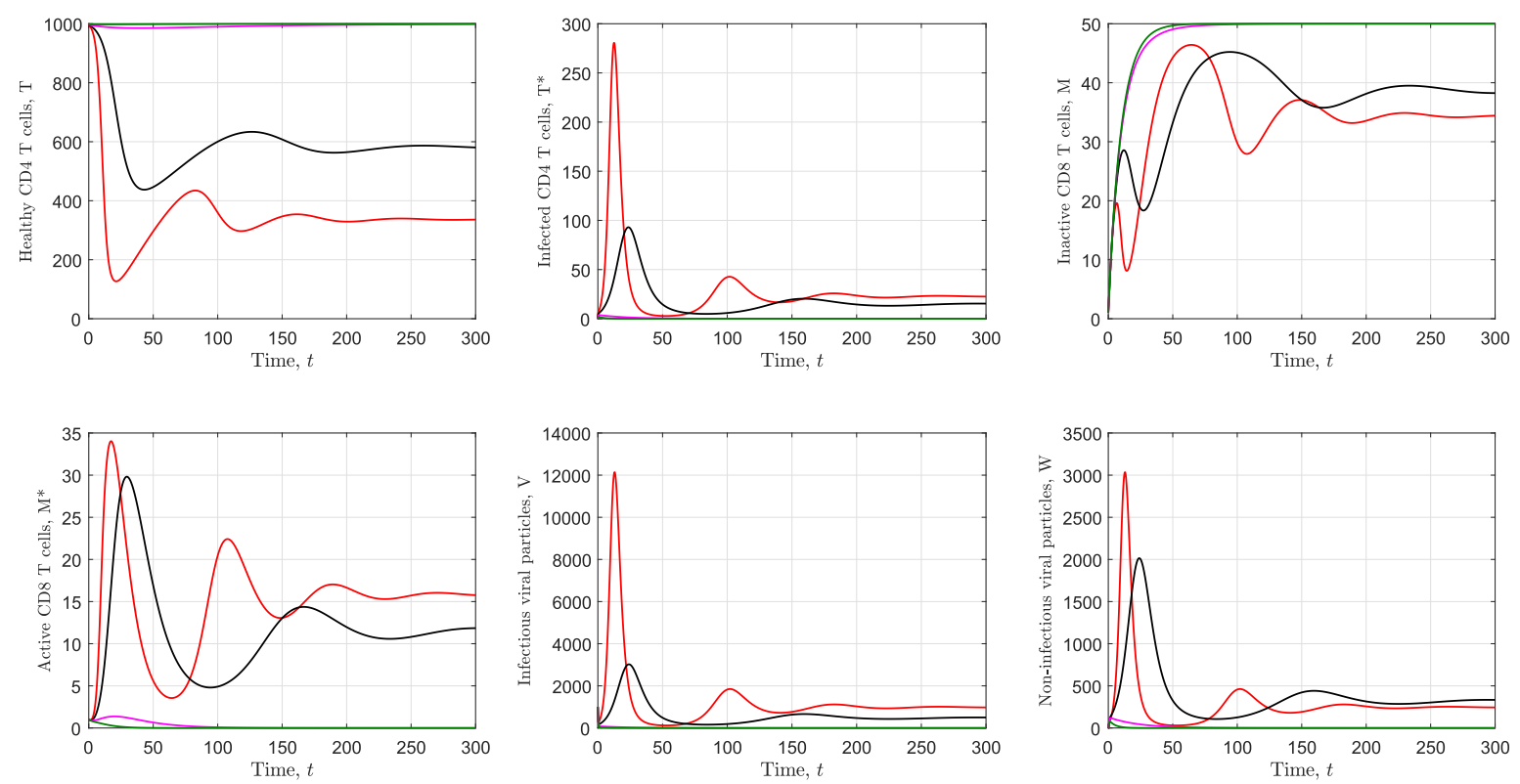

Figura 6. Numerical solutions of system (7) considering the parameters from Table $2, \gamma=2 \times 10^{-3}$, $\beta=2.5 \times 10^{-5}$, and varying the values for the ART effectiveness, under the assumption of being $u_{1}=u_{2}$. The red curve represents $u_{i}=0.2$, where $R_{0}=1.2979$, the black curve corresponds to $u_{i}=0.4$, with $R_{0}=0.7301$, the magenta curve to $u_{i}=0.6$ and $R_{0}=0.3245$, and the green curve is $u_{i}=0.8$ with $R_{0}=0.0811$, for $i=1,2$.

are achieved. Once again, these results permit validating the model, evidencing that the use of different inhibitors in combination is more convenient than using them in isolated manner.

\subsection{Coupling algorithm of the immunological and populational scales}

For the purpose of coupling the model in the immunological scale (system of differential equations) with the model for the propagation in the populational scale (complex network), an algorithm was designed that permits manipulating the complex network, so that events in the populational scale, that is, the way the virus will spread in the in the population, are influenced by that which occurs within the immune system of each individual.

1. The network was constructed for the population beginning with the random values shown in the following intervals [31-33], which seeks for each individual $i$, to $i=1, \ldots, N_{j}$, from the network to have a different immune system. Additionally, for the values of the other parameters used in the system of differential equations that describes the immunological scale, those described in the Table 2.

a) Initial number of healthy CD4 T cells in the $i$-th person per $\mathrm{mm}^{3}: T_{i}(0) \in[200,1000]$.

b) Initial number of inactive CD8 $\mathrm{T}$ cells in the $i$-th person per $\mathrm{mm}^{3}: M_{i}(0) \in[70,980]$.

c) Values of infected CD4 T cells, active CD8 T cells, infectious and non-infectious viral particles will be null, given that it is considered that the $i-$ th person is still not infected by the virus, that is, $T_{i}^{*}(0)=M_{i}^{*}(0)=V_{i}(0)=W_{i}(0)=0$.

d) Production rate of healthy cells of the $i$-th person: $\sigma_{i} \in[5,20]$.

e) Infection probability of the healthy cells of the $i$-th person: $\beta_{i} \in\left[0,2.5 \times 10^{-5}\right]$.

f) Production rate of viral particles of the $i$-th person: $N_{i} \in[100,1500]$.

Then, a vertex from the network is chosen randomly as the first infected and is assigned a viral load between 1 and $1000 \mathrm{vir} / \mathrm{mm}^{3}$ [34]. With the aforementioned, the network is created, obtaining the initial state for $\tau=0$.

2. From the corresponding initial condition, we solve the system of differential equations of the immunological system (7) for each vertex $i$ during one unit of time, saving the numerical solutions $T_{i}(t), T_{i}^{*}(t), M_{i}(t), M_{i}^{*}(t), V_{i}(t)$ and $W_{i}(t)$, therefore, we have the immunological status of each 


\begin{tabular}{||l|c|c||}
\hline Type of risk & viral particle value & infection probability \\
\hline \hline Low risk & $V_{i}<200$ & $\lambda_{i} \in(0.0006,0.0011)$ \\
Medium risk & $200 \leq V_{i}<2000$ & $\lambda_{i} \in(0.0007,0.0168)$ \\
High risk & $V_{i} \geq 2000$ & $\lambda_{i} \in(0.002,0.025)$ \\
\hline
\end{tabular}

Cuadro 3. Values for $\lambda_{i}$ according to the number of vir $/ \mathrm{mm}^{3}$ found in the immune system [33].

individual in the population during that unit of time. Additionally, the final value of each of the numerical solutions will be used as the initial condition in the following iteration. Also, we save the number of susceptibles, carriers with therapy and carriers without therapy at the end of the iteration.

3. Based on the numerical solutions, the following decisions are made:

a) If the $i$-th person is an HIV carrier and presents a number of healthy CD4 T cells $T_{i}$ below $350 \mathrm{cell} / \mathrm{mm}^{3}$, a random probability of accessing to ART is assigned, if it is above 0.6, it is established to use ART, so that a random value is assigned for $u_{1}$ and $u_{2}$ between $(0,1)$.

$b$ ) If the $i$-th person is HIV carrier already using ART, a random probability of therapy abandonment is assigned and if it is below 0.2 , it is established that the individual leaves ART, that is $u_{1}=u_{2}=0$.

This is why in the simulations that will be presented ahead in different iterations, the state of the vertices will vary going from carriers with therapy to carriers without therapy and vice versa.

4. The transmission process considers different factors. If the $i$-th person is HIV carrier, the decision is made randomly to have or not a sexual encounter with only one of their susceptible "neighbors" (another susceptible vertex sharing an edge). If the random decision was affirmative, the infected person is partnered with one of their susceptible sex partners, then, the probability of transmitting the infection from the infected person $i$ to the susceptible person $j$ is given by $\lambda_{i j}=\frac{i j \lambda_{i}}{\langle k\rangle}$ (taken from [35]), where $\langle k\rangle$ represents the average degree associated with the potential number of sex partners and $\lambda_{i}$ is the infection probability through sexual contact; the probability $\lambda_{i}$ is taken from [33] and is shown in the Table 3 according to the values of vir $/ \mathrm{mm}^{3}$.

Finally, if the person $j$ becomes infected, an initial viral load is assigned with $10 \%$ of the load owned by the person $i$ who caused the infection.

5. At the end of each iteration, prevalence was calculated, as the rate between the number of HIV carriers with or without therapy and the total number of people from the network, that is,

$$
P_{f}=\frac{\sum y_{i}+\sum z_{i}}{\sum x_{i}+\sum y_{i}+\sum z_{i}} .
$$

The algorithm described is conducted until $\tau=\tau_{f}$, the total number of iterations.

\subsection{Simulation of the coupled model}

In order to illustrate the coupled model, a simulation shown in Figure 7 was performed for $N_{j}=25$ people, $k=2$, and $\tau_{f}=20$ iterations. Additionally, two more simulations are shown for $N_{j}=1000$, $\tau_{f}=200, k=4$ (Figure 9 ) and $k=10$ (Figure 10). Those simulations illustrate the implementation of the model and do not intend to reflect real scenarios. In each case, the blue vertices represent people susceptible of acquiring HIV $x_{i}$, those in red represent HIV carriers without therapy $y_{i}$, and those in green represent HIV carriers who use ART $z_{i}$.

Figure 7 shows the initial network, iterations $2,6,10,11,12,13,14,17$, and the final network; hence, it shows the infection's spread over time, obtaining a final prevalence $\left(P_{f}\right)=0.68$. The first vertex infected is 19, at iteration 6 it manages to infect vertex 8 . Then, at iteration 10 the number of HIV carriers has increased because vertices 2 and 5 are the new ones infected. Later, at iteration 14, the number of HIV carriers is seven (vertices 1, 2, 5, 8, 9, 19, and 21). Lastly, the network ends 

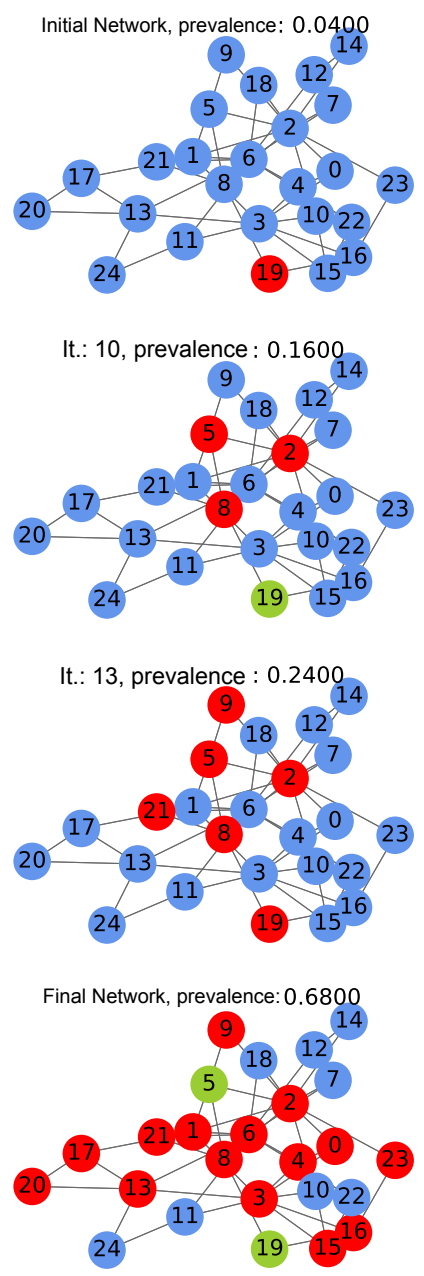
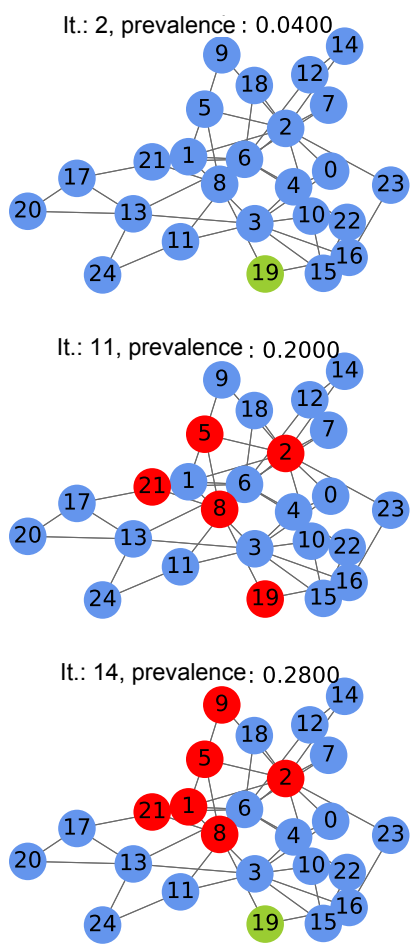
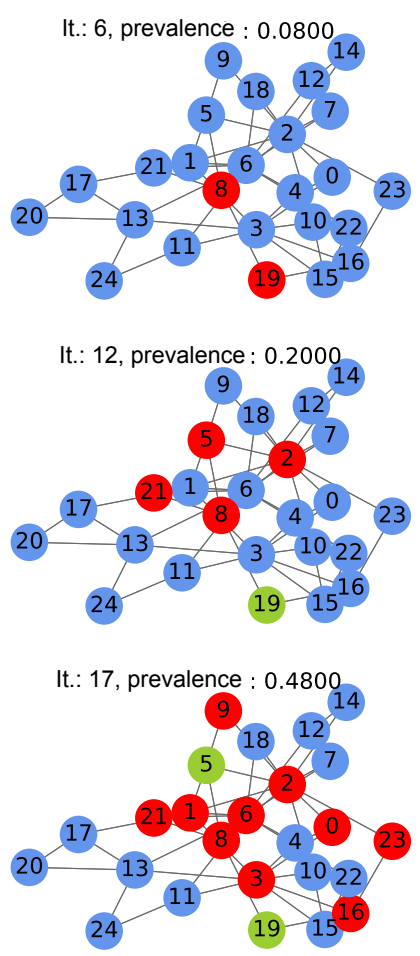

Figura 7. Schematic representation of the network showing the evolution with respect to time for $N_{j}=25$, $\tau=20, \gamma=3.4$, and $k=2$. The blue vertices represent the people susceptible of acquiring the infection, those in red the carriers without ART, and those in green the carriers who use ART. The scenario displayed does not seek to represent reality, it only aims to illustrate the operation of the coupled model.

with 17 people infected, from which two are using ART (vertices 5 and 19 in green). Note that during the simulation time, vertex 19 accessed therapy and abandoned it on several occasions (in iterations 2 , 10, 12, and 14 vertex 19 accessed ART and abandoned it in iterations 6, 11, and 13). Vertex 5 was without therapy and accessed it in iteration 17. As already mentioned, the numerical solutions of each person's immune system were saved for each iteration of the network, hence, Figure 8 shows the cell populations $T, T^{*}, M, M^{*}$ and viral loads $V$ and $W$ of individuals $1,8,19$, and 24 of the network simulated in Figure 7, as an example. Black curves means the person remained susceptible during the entire simulation time; red represents a person who is an HIV carrier and who in the final time $\tau_{f}$ did not use ART; lastly, green represents a person who is an HIV carrier and who in the final time $\tau_{f}$ uses therapy. Note that each person began with a different number of $T$ and $M$ cells, which guarantees that a different immune system is considered for each one (numeral 1 of the previous subsection).

Simulation presented in Figure 8 shows the immune system of person 19, who was the first infected, upon ending the simulation time this person used ART (green). In the curves of viral particles $V$ and $W$ it may be estimated that ART was begun since iteration 2, but it is seen that therapy is alternatively taken and interrupted form iteration 10, which is revealed by the oscillations (high peaks) shown in both curves. As expected, when abandoning ART, HIV manages to replicate and high levels of viremia are reached. Furthermore, it is observed that person 19, during most of the simulation time, maintained low levels of healthy cells $T$, below 360 cell $/ \mathrm{mm}^{3}$. Person 8 acquired the virus during an early stage of 

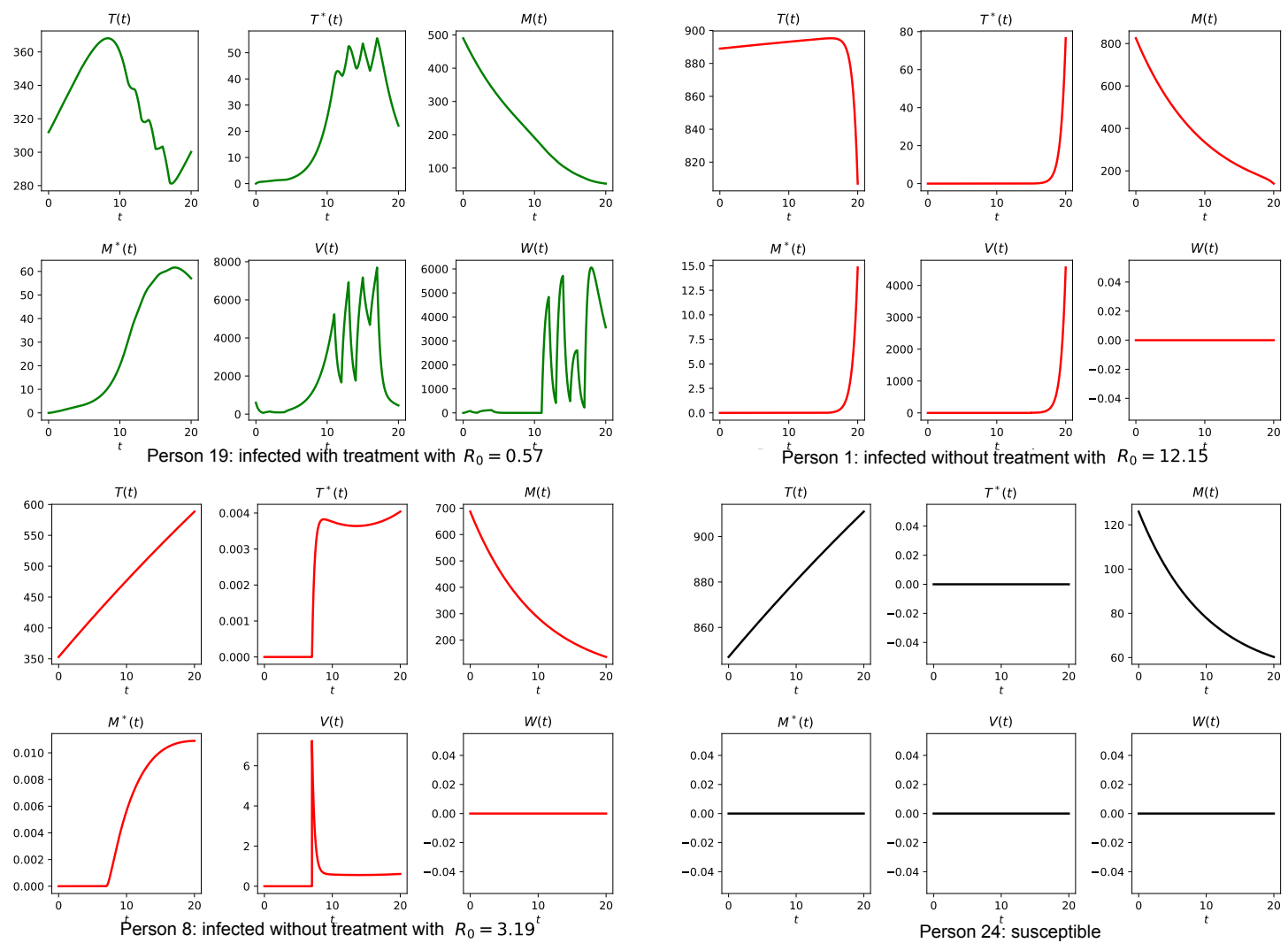

Figura 8. Immunological state of individuals $1,8,19$, and 24 of the complex network simulated with $N_{j}=25, \tau=20, \gamma=3.4$ and $k=2$. (Black: person susceptible throughout the simulation time. Red: HIV carrier who in the final time did not use ART. Green: HIV carrier who in the final time uses therapy).

the simulation (iteration 6 ) this is why for $\tau=7$ its $T^{*}$ and $M^{*}$ cells and viral particles $V$ began to reach high levels, also notice that this person started using RTI at iteration 9 but never used the PI during the simulation time, which is why the $W$ values remain at zero; upon ending the simulation time, this person did not use ART. It is also observed that, although person 8 is infected, the number of healthy cells $T$ did not diminish at any moment during the simulation period, a situation that possibly would change if we increase the number of iterations. Person 1 did not use the PI at any moment of the simulation, which is why the number of non-infectious viral particles $W$ remained at zero; moreover, it is also noted that this person acquired the infection almost at the final of the simulation (iteration 14), but in spite of that achieved an $R_{0}=12.15$, it could be inferred that, upon increasing the number of iterations, and if this person remains without using ART, the virus spread will be accelerated, generating an infection outbreak and which would cause a much greater probability of infecting their susceptible sex partners and of the virus spreading faster in the population. In deed, although person 1 was infected in the almost final stage, its infectious viral particles $V$ reached high values (note the high value of $R_{0}$ ). Finally, Person 24 is susceptible, hence, during the simulation time was never infected with HIV and this is the cause for only $T$ and $M$ cell populations having values different from zero.

Figure 9 shows the simulation for a bigger population with $N_{j}=1000$, a higher number of iterations $\tau_{f}=200$, and takes $\gamma=3.4$ from the literature [16,22]. This simulation refers to a slightly conservative population, given that it has been assigned a maximum number of sex partners of $k=4$. Figure 9 shows the initial network with the first vertex infected (red upper left panel), the final network (upper right panel), the plot showing the population of HIV carriers who were using ART (green dots) and those that did not use it (red dots) located on the lower left panel and finally the prevalence (lower right panel). The plot for both HIV carrier populations with and without therapy is constructed by accounting the number of people in the different states (susceptible, infected with ART, infected 
Initial Network, prevalence: 0.0010

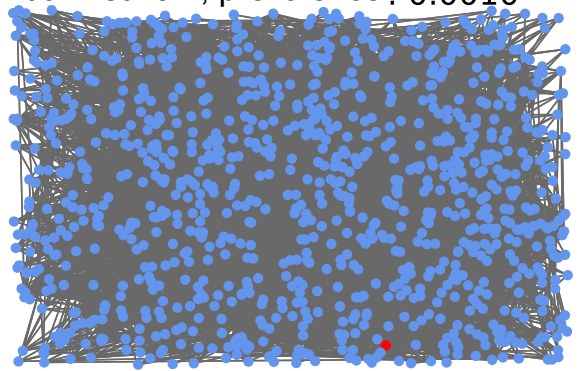

People infected with ART and without ART

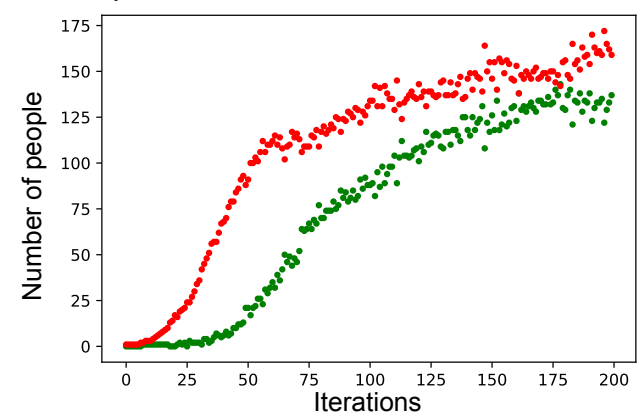

Final Network, prevalence: 0.2960
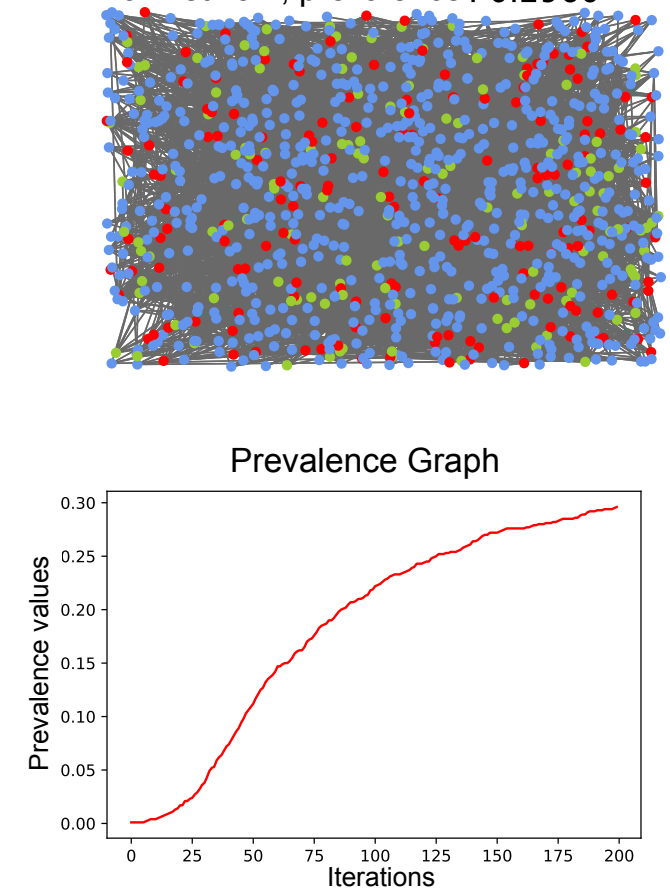

Figura 9. Superiores: Simulation of the complex network for $N_{j}=1000, \tau=200, \gamma=3.4$, and $k=4$ (blue: susceptible people, red: carriers without ART, green: carriers with ART). lower left: Graphic showing the relation between the HIV carrier population with ART (green) and the carrier population without ART (red). lower right: Graphic of virus prevalence in the population. The scenario shown does not seek to represent reality, it only aims to illustrate the operation of the coupled model.

without ART) in each iteration; we obtained, as a result, sequences of points that tended to stabilize over time, with the red curve being greater or equal to that of the green most of the time, which means that $y_{i}<z_{i}$, It may be inferred from the plot, that both populations tend to stabilize at an equilibrium value: for the first, that equilibrium value is around the 160 people, that is, $16 \%$ of the HIV carriers uses ART at the end of simulation, while for the population of HIV carriers without ART, the equilibrium point is around 135 people, that is, $13.5 \%$ of the HIV carrier population does not use ART at the end of the simulation. This situation is repeated in all the simulations we performed, despite here we are assuming the probability of accessing ART as 0.6 and the probability of abandoning ART as 0.2, This is an interesting result indicating that the number of people who do not access ART being higher than those who access ART is an interesting source of information that should be studied in more detail. The prevalence plot (lower-right panel) provides an approximate idea of when the virus spread may have started. In this case, it is observed that it was close to iteration 5 . The prevalence values are found through the equation (10) computed at each iteration. It is necessary to highlight that the prevalence plot grows in its entire domain and this is due to HIV not having a cure; hence, a virus carrier, with or without therapy, cannot go on to become a susceptible person; in the simulation, the final prevalence value was 0.2960 .

Figure 10 shows the simulation of a more liberal population, where the maximum number of sex partners is $k=10$, taking the same amount of people for the population and the same number of iterations, that is, $N_{j}=1000$ and $\tau_{f}=200$, respectively, and $\gamma=3.4$ was again taken from the literature [16,22]. Figure 10 also shows the initial network with its first infected (upper left panel), the final network (upper right panel), the graphic showing the relation between people infected with and without ART (lower left panel), and the prevalence graphic (lower right panel). In the final network a prevalence of 0.4950 is achieved, which is quite high and means that $49 \%$ of the population is HIV carrier. This simulation shows a scenario where people have a large number of sex partners and use no protection. In comparison with the simulation shown in Figure 9, it is evidenced that values of $k$ 
Initial Network, prevalence: 0.0010

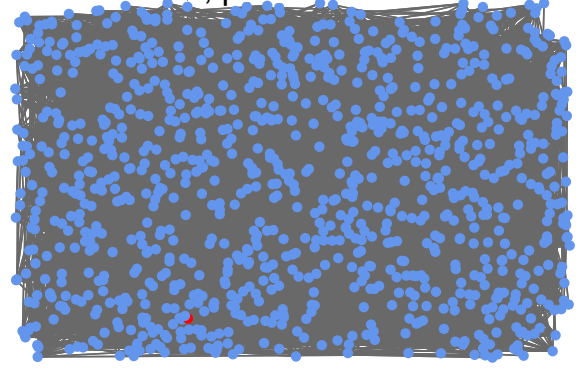

People infected with ART and without ART

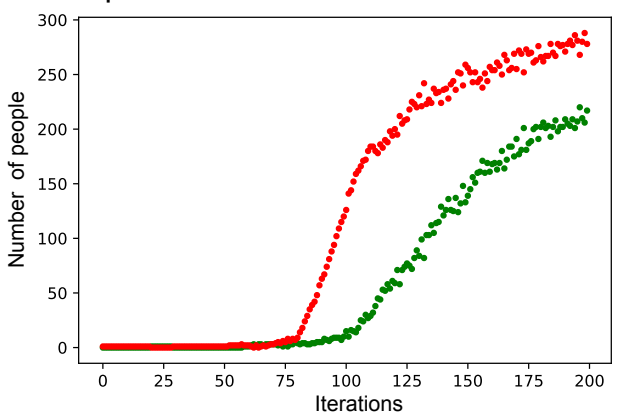

Final Network, prevalence : 0.4950
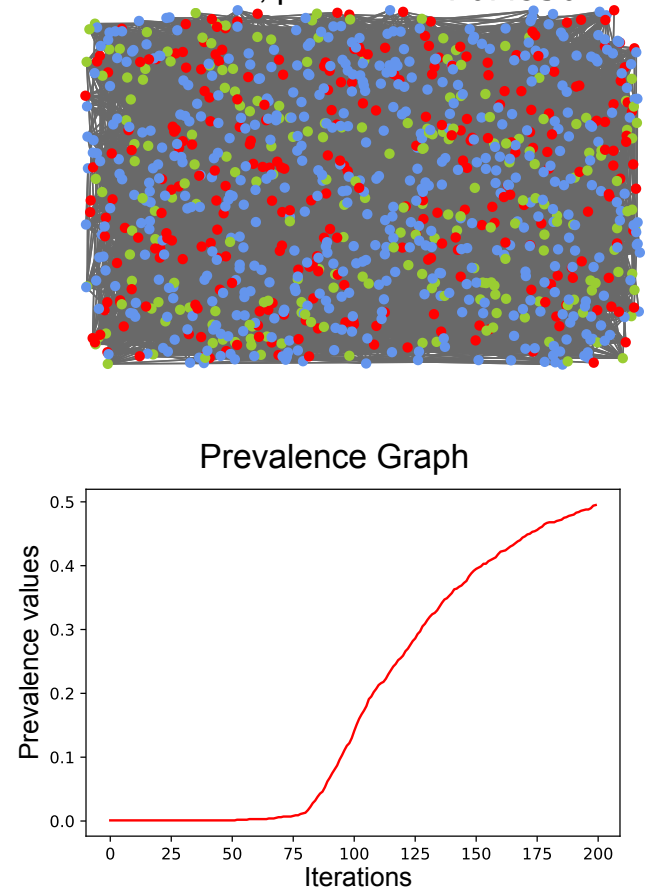

Figura 10. Superiores: Simulation of the complex network for $N_{j}=1000, \tau=200, \gamma=3.4$, and $k=10$ (Blue: susceptible individuals. Red: carriers without ART. Green: carriers with ART). lower left: Graphic showing the relation between people infected with and without ART. lower right: Graphic of virus prevalence over time $\tau$. The scenario shown does not seek to represent reality, it only aims to illustrate the operation of the coupled model.

cause the network to become susceptible to changes in the rate with which the virus spreads. Due to the aforementioned, the infection prevalence in the slightly conservative population with $k=4$ was lower than that obtained in the more liberal population with $k=10$. Analogous to the previous simulation, for the graphic showing the relation between the population of carriers not using ART and those who use it in lower left panel, the population not using ART continues being more numerous during the entire simulation time; besides, this population tends to stabilize over time at values close to 270 people approximately, this is $27 \%$ of the total population, unlike the population of people infected who use ART in which the equilibrium value is reached around 210 people, that is, $21 \%$ of the 1000 people; again, this evidences that the number is higher of people who do not have access to ART. The prevalence plot shows that close to iteration 50, virus spread begins to accelerate, the curve growth is more pronounced (greater slope) and, finally, the curve starts reducing (the slope diminishes) towards the end indicating a fast virus spread followed by a slow propagation, it is also noted that this plot presents an equilibrium point, which coincides with the prevalence value in the final time $\tau_{f}$. Although in the simulation for a conservative population the infection outbreak occurred in time $\tau=5$, the prevalence was lower than the simulation made for a more liberal population, where the infection outbreak took place over later time, for $\tau=50$.

\section{Conclusions}

This paper studies a multiscale model for HIV propagation in a group of people between 15 and 24 years of age, through a cluster-type scale-free complex network, which follows a power law. The model relates the immunological scale with the populational scale so that the information obtained from the immunological scale helped to model decision making on how the infection would spread in the population.

The infection is controlled when reducing the probability that healthy CD4 $\mathrm{T}$ cells become infected. Thereby, when small values are achieved in said probability, the infection remains in low levels. It was 
also evidenced that when the cellular immune response has a high probability of eliminating infected cells, the infection can be controlled; essentially, these results validate the model since they correspond with known facts about the infection process and the specific cell immune response to HIV. The model analysis reflect how the use of reverse transcriptase inhibitors (RTI) and protease inhibitors (PI) help to control the infection in the immune system of each HIV carrier, as well as in the spread of the infection within the population. Therapies help to reduce outbreaks of the infection and evidence that when using different types of ART at the same time better results are achieved than using them in isolated manner, which again validates the model for the immunological scale studied here. Lastly, with the assumptions of the model of the immunological scale and the values of the parameters used in the simulations, it was observed that when reaching ART effectiveness in the interval $(0.8,1)$, the viral load becomes undetectable and large values are reached for the healthy CD4 T cells.

Different scenarios are found in the simulations of the immune system for the populational scale. The first deals with people who are not infected during the entire simulation period, which is why there are only values in the number of healthy cells $T$ and inactive cells of the cellular response $M$, for the other variables, populations are shown in zero. Another scenario is that shown by people who use ART, but only use the reverse transcriptase inhibitors, which is why the non-infectious viral particles $W$ is shown in zero, given that the PI were not used, these particles were not produced. Also, a very characteristic scenario was found, that of HIV carriers who take and abandon therapy on various occasions during the simulation time, which is why we get graphics with maximums and minimums in forms of peaks. Finally, another scenario related with $R_{0}$ is that which shows that when large values are obtained $(>1)$, the infection spreads faster in the organism of each carrier, unlike low $R_{0}$, which keep the HIV from propagating quickly, or that (theoretically) the person is not infected when $R_{0}<1$, scenarios that can be associated with undetectable viral loads.

Moreover, it is observed from simulations that a higher number of sex partners a person has within a specific population, means a higher prevalence of the virus within said population and that the infection will spread at higher rates, as long as the sexual encounters are without protection. Additionally, the simulations that relates the number of HIV carriers with and without ART, we obtained as a result sequences of points that tended to stabilize over time, and in all the cases simulated the population of HIV carriers without therapy was greater or equal to those with therapy. This reflects an interesting relation between access and abandonment of ART, which is something we cannot explain based on the parameters considered here, since the model takes the probability of access of 0.6 and the probability of abandonment of 0.2 . Further studies should be performed in this regard. Nevertheless, our coupling algorithm of the immunological scale with the populational scale, takes random probabilities for access and abandonment of ART for each HIV carrier at each iteration, thus, we consider that for future research the most adequate would be to assign these values in non-random manner, using the viral load and the CD4 T cell count.

In regard to prevalence, it is not necessary, that to obtain large values, to start viral propagation during early stages of the simulation time. This is due to many factors that influence decision making on the way of virus spread, it may occur that although the first person is infected over early time of simulation, small values are obtained in prevalence; thus, it may be inferred that much uncertainty exists when not knowing how the virus will spread and how much impact it will have in a population. In addition, the prevalence tends to equilibrium point, which is equal to the prevalence value in the final time. Given that although equal values are used for the parameters, each simulation will lead to different prevalence results and a different virus propagation behavior, it is suggested that a future works conduct multiple simulations with the same parameters to statistically infer the prevalence expected value for those parameters.

Finally, it is believed that the formulation of this mathematical model is correct, since taking into account the two scales studied (immunological and population), it reflects the assumptions that are made about HIV. In deed, the multiscale model studied allows us to give an overview of what the dynamics of infection would be like in a population, if the immune status of the people within said 
population is taken into account, it can also serve as a basis for future research that seeks to consider more variables in order to achieve a model closer to reality. It is necessary to clarify that the values used and the resulting data do not illustrate a real picture, since just comparing the real data worldwide, there are around 7.900 million people [37] in the world and if we have into account the number of people infected with HIV worldwide [3], the prevalence will result in approximately 0.005. However, models of this nature would allow us to explore the spread of HIV in population groups with specific risks in greater detail.

Author Contributions: Conceptualization, H.D.T.-Z. and S.A.V.-Q.; methodology, H.D.T.-Z.; software, H.D.T.-Z. and S.A.V.-Q.; validation, H.D.T.-Z., S.A.V.-Q. and D.A.P-M.; writing: original draft, S.A.V.-Q.; writing: revision and editing, H.D.T.-Z., S.A.V.-Q. and D.A.P-M.; supervision, H.D.T.-Z.

Funding: This research was funded by University of Quindío through the research project 922

Acknowledgments: H. D. Toro-Zapata and S.A.Vásquez-Quintero were supported by Universidad del Quindío, through the research project 922 .

Conflicts of Interest: The authors declare having no conflicts of interest.

\section{Abbreviations}

The following abbreviations were used in this research work:
HIV Human Immunodeficiency Virus
AIDS Acquired immune deficiency syndrome
ART Antiretroviral therapy
RTI Reverse transcriptase inhibitors
PI Protease Inhibitors
LAE locally asymptotically stable

\section{References}

1. AIDS InfoNet. http://www.aidsinfonet.org/uploaded/factsheets/204_spa_485.pdf (Recovered on 22 May 2018).

2. Organización Mundial de la Salud. https://www.who.int/features/factfiles/hiv/es/ (Recovered on 14 February 2019).

3. ONUSIDA. https://www.unaids.org/sites/default/files/media_asset/UNAIDS_FactSheet_es.pdf (Recovered on 29 September 2020).

4. InfoSIDA. https://www.infosida.es//que-es-el-vih (Recovered on 26 March 2018).

5. Planas, G. La infección por el virus de la inmunodeficiencia humana. In Farmacia Hospitalaria Tomo II, 3rd ed.; Fundación Española de Farmacia Hospitalaria: Madrid, España, 2002; pp. 1493-1516.

6. Dirección general de comunicación por los derechos humanos de la CDHDF. VIH un llamado a la acción positiva. Dfensor 2014, 2, 5-19.

7. Velasquez S.; Bedoya,B. Los jóvenes: población vulnerable del VIH/SIDA. MEDICINA UPB 2010, 29, 144-154.

8. ONUSIDA. http://data.unaids.org/topics/young-people/youngpeoplehivaids_es.pdf (Recovered on 25 May 2018).

9. Comisión Nacional de los Derechos Humanos México. https://www.cndh.org.mx/sites/default/files/doc/ Programas/VIH/Divulgacion/cartillas/Cartilla-DH-Jovenes-VIH-Sida.pdf (Recovered on 14 April 2018).

10. Instituto Nacional de Salud. Boletín Epidemilógico Semanal, Semana epidemiológica 47, 17 al 23 de noviembre de 2019. https://www.ins.gov.co/buscador-eventos/BoletinEpidemiologico/2019_Boletin_ epidemiologico_semana_47.pdf (Recovered on 29 September 2020).

11. NorthShore University HealthSystem https://www.northshore.org/healthresources/encyclopedia/ encyclopedia.aspx?DocumentHwid=tm6421\&Lang=es-us (Recovered on 27 July 2018).

12. ONUSIDA. https://www.unaids.org/sites/default/files/media_asset/JC2484_therapy-2015_es_1.pdf (Recovered on 7 January 2018).

13. Zapata, H. D. T.; Vasquez, E. R.; Mazo, M. J. M. Modelo estocástico para la infección con VIH de las células CD4 T+ del sistema inmune. Revista de Matemática: Teoría y Aplicaciones 2017, 24(2), 287-313. 
14. Toro, H. D.; Rodríguez, A. J. O.; Medellín, D. A. P. Modelo para el acceso efectivo al tratamiento antirretroviral en relación con el fracaso terapéutico de la infección por VIH. Revista EIA 2019, 16(31), 115-130.

15. Toro H.; Caicedo A.; Lee S. The Role of Immune Response in Optimal HIV therapy Interventions. Processes 2018, 6(102), pag 1-16.

16. Newman, M. E.; Barabási, A. L. E.; Watts, D. J. The structure and dynamics of networks; Princeton university press: Nueva Jersey, Estados Unidos, 2006; pp. 1-8.

17. Barabási, A. L.; Albert, R. Emergence of scaling in random networks. Science 1999, 286(5439), 509-512.

18. Networkx. https://networkx.github.io/documentation/networkx-1.10/reference/generated/networkx. generators.random_graphs.powerlaw_cluster_graph.html\#networkx.generators.random_graphs. powerlaw_cluster_graph (Recovered on 02 September 2019).

19. Uruguay. Ministerio de Salud Pública. Dirección General de la Salud. Departamento de Programación Estratégica en Salud. Área Salud Sexual y Reproductiva (2017). Programa Nacional ITS-VIH/Sida. Infección por virus de la Inmunodeficiencia Humana (VIH): pautas para diagnóstico, monitorización y tratamiento antirretroviral. Actualización 2014.

20. InfoSIDA. Glosario del VIH. https:/clinicalinfo.hiv.gov/themes/custom/aidsinfo/documents/ spanishglossary_sp.pdf (Recovered 3 October 2020).

21. Sloot, P. M.; Ivanov, S. V.; Boukhanovsky, A. V.; van de Vijver, D. A. \& Boucher, C. A. Stochastic simulation of HIV population dynamics through complex network modelling. International Journal of Computer Mathematics 2008, 85(8), 1175-1187.

22. Instituto de Ciencias Físicas (ICF) de la UNAM. https://www.fis.unam.mx/ max/English/notasredes.pdf (Recovered on 20 March 2019).

23. infoSIDA, (2018). Glosario de InfoSIDA. Edición 9A.

24. Bubnoff A. Juego de clones. VAX 2013, 11(5), 2-5.

25. Culshaw R.; Ruan S.; Spiteri R. Optimal hiv therapy by maximising immune response. J. Math. Biol. 2004, 48,545-562.

26. Orellana J.M. Optimal drug scheduling for HIV therapy efficiency improvement. Biomed. Signal Process. Control 2011, 6,379-386.

27. Ramírez, C.; Muñoz, A.; García, M. Modelos Biomatemáticos II. Ediciones Uniquindio 2008.

28. Londoño González, C. A.; Toro Zapata, H. D.; Trujillo Salazar, C. A. Modelo de simulación para la infección por VIH y su interacción con la respuesta inmune citotóxica. Revista de Salud Pública 2014, 16,103-115.

29. Toro, H. Z.; Caicedo, A. C.; Bichara, D.; Lee, S. Role of active and inactive cytotoxic immune response in human immunodeficiency virus dynamics. Osong public health and research perspectives 2014, 5(1),3-8.

30. Toro Zapata, H. D.; Trujillo Salazar, C. A.; Prieto Medellín, D. A. Evaluación teórica de estrategias óptimas y sub-óptimas de terapia antirretroviral para el control de la infección por VIH. Revista de Salud Pública 2018, 20,117-125.

31. Noda Albelo, A. L.; Vidal Tallet, L. A.; Pérez Lastre, J. E.; Cañete Villafranca, R. Interpretación clínica del conteo de linfocitos CD4 T positivos en la infección por VIH. Revista Cubana de Medicina 2013, 52(2), 118-127.

32. Avila, L. M.; Gómez, C. P.; Ríos, R. S.; Laverde, E. Estandarización de valores normales de linfocitos CD3, CD4, CD8 y relación CD4/CD8 por citometría de flujo en individuos sanos colombianos. Acta Médica Colombiana 1996, 21(4), 158-161.

33. Lasry, A.; Sansom, S. L.; Wolitski, R. J.; Green, T. A.; Borkowf, C. B.; Patel, P.; Mermin, J. HIV sexual transmission risk among serodiscordant couples: assessing the effects of combining prevention strategies. Aids 2014, 28(10), 1521-1529.

34. GeoSalud. https://www.geosalud.com/vih-sida/analisis_sangre_pg5.htm (Recovered on 10 August 2019).

35. May, R. M.; Lloyd, A. L. Infection dynamics on scale-free networks. Physical Review E 2001, 64(6), 066112.

36. Wiggins S., (2003). Introduction to Applied Nonlinear Dynamical Systems and Chaos. Second Edition. Springer-Verlag, New York.

37. Worldometer. Población mundial. Taken from https://www.worldometers.info/es/ on 02 November 2020. 
Appendix: demonstration of propositions and basic reproduction number

Proof of Proposition 1. Starts by demonstrating that for each coordinated hyperplane of $\mathbb{R}^{6}$ (if the initial conditions are non-negative and considering that $0 \leq u_{i} \leq 1$ by definition, with $\left.i=1,2\right)$ the solutions are found in the non-negative octant of $\mathbb{R}^{6}$, in effect:

$$
\begin{aligned}
& \left.\dot{T}\right|_{T=0}=\sigma-\beta\left(1-u_{1}\right)(0) V-\mu(0)=\sigma>0 \\
& \left.\dot{T}^{*}\right|_{T^{*}=0}=\beta\left(1-u_{1}\right) T V-\gamma(0) M^{*}-\delta(0)=\beta\left(1-u_{1}\right) T V \geq 0 \\
& \left.\dot{M}\right|_{M=0}=\lambda-\psi T^{*}(0)-\rho(0)=\lambda>0 \\
& \left.\dot{M}^{*}\right|_{M^{*}}=\alpha T^{*}(0)-\rho(0)+\psi T^{*} M=\psi T^{*} M \geq 0 \\
& \left.\dot{V}\right|_{V=0}=N \delta\left(1-u_{2}\right) T^{*}-c(0)=N \delta\left(1-u_{2}\right) T^{*} \geq 0 \\
& \left.\dot{W}\right|_{W=0}=N \delta u_{2} T^{*}-c(0)=N \delta u_{2} T^{*} \geq 0
\end{aligned}
$$

Now it is guaranteed that the solutions are bound superiorly. For $V=0$ in (1) we obtain:

$$
\dot{T} \leq \sigma-\mu T \Longleftrightarrow \dot{T}+\mu T \leq \sigma .
$$

Then, the inequality is solved, yielding as result $T(t) \leq \frac{\sigma}{\mu}+\frac{k}{e^{\mu t}}$, hence $\lim _{t \rightarrow \infty} T(t) \leq$ $\lim _{t \rightarrow \infty}\left(\frac{\sigma}{\mu}+\frac{k}{e^{\mu t}}\right)=\frac{\sigma}{\mu}$. Thus, $T \leq \frac{\sigma}{\mu}$.

Also, if $G=T+T^{*}+M+M^{*}$, then:

$$
\begin{aligned}
\dot{G}= & \sigma-\beta\left(1-u_{1}\right) T V-\mu T+\beta\left(1-u_{1}\right) T V-\gamma T^{*} M^{*}-\delta T^{*}+ \\
& \lambda-\psi T^{*} M-\rho M+\alpha T^{*} M^{*}+\psi T^{*} M-\rho M^{*} \\
= & \sigma-\mu T-\gamma T^{*} M^{*}-\delta T^{*}+\lambda-\rho M+\alpha T^{*} M^{*}-\rho M^{*} \\
= & \sigma+\lambda+\alpha T^{*} M^{*}-\gamma T^{*} M^{*}-\left(\mu T+\delta T^{*}+\rho M+\rho M^{*}\right) \\
\leq & \sigma+\lambda+(\alpha-\gamma) T^{*} M^{*}-\varepsilon\left(T+T^{*}+M+M^{*}\right) ; \operatorname{con} \varepsilon=\operatorname{máx}\{\mu, \delta, \rho\} \\
\leq & \sigma+\lambda-\varepsilon G+(\alpha-\gamma) T^{*} M^{*}, \operatorname{supposing} \gamma \geq \alpha \\
\leq & \sigma+\lambda-\varepsilon G-(\gamma-\alpha) T^{*} M^{*} \\
\leq & \sigma+\lambda-\varepsilon G .
\end{aligned}
$$

Through a process analogous to that used to narrow down the $T$ variable, we obtain that $G(t) \leq \frac{\sigma+\lambda}{\varepsilon}$, hence, particularly $T^{*}(t) \leq \frac{\sigma+\lambda}{\varepsilon}$

Finally, if $D=V+W$, then:

$$
\begin{aligned}
\dot{D} & =N \delta\left(1-u_{2}\right) T^{*}-c V+N \delta u_{2} T^{*}-c W \\
& =N \delta T^{*}\left(1-u_{2}+u_{2}\right)-c(V+W) \\
& =N \delta T^{*}-c D \\
& \leq N \delta \frac{\sigma+\lambda}{\varepsilon}-c D \\
& \leq \frac{N \delta(\sigma+\lambda)}{\varepsilon c} .
\end{aligned}
$$

It is then obtained that $D(t) \leq \frac{N \delta(\sigma+\lambda)}{\varepsilon c}$, with which the test is finished.

\section{Basic reproduction number $R_{0}$}

To find the $R_{0}$ of the model (7), we define vectors $h$ and $g$ corresponding to the equations of the populations responsible for the infection: 
22 of 25 


$$
h=\left(\begin{array}{c}
\beta\left(1-u_{1}\right) T V-\gamma T^{*} M^{*} \\
0
\end{array}\right) \quad g=\left(\begin{array}{c}
-\delta T^{*} \\
N \delta\left(1-u_{2}\right) T^{*}-c V
\end{array}\right)
$$

The next-generation matrix is defined as $\tilde{M}=H G$, where $H=-D h$ and $G=(D g)^{-1}$. For $D h$ and $D g$, the respective Jacobian matrices of $h$ and $g$ with respect to $T^{*}$ and $V$.

Now, we proceed to calculate $G$ :

$$
H=-\left(\begin{array}{cc}
\frac{\partial h}{\partial T^{*}} & \frac{\partial h}{\partial V} \\
0 & 0
\end{array}\right)=\left(\begin{array}{cc}
\gamma M^{*} & -\beta\left(1-u_{1}\right) T \\
0 & 0
\end{array}\right)
$$

$$
G=\left(\begin{array}{cc}
\frac{\partial g}{\partial T^{*}} & \frac{\partial g}{\partial V} \\
\frac{\partial g}{\partial T^{*}} & \frac{\partial g}{\partial V}
\end{array}\right)^{-1}=\left(\begin{array}{cc}
-\frac{1}{\delta} & 0 \\
-\frac{N \delta\left(1-u_{2}\right)}{\delta c} & -\frac{1}{c}
\end{array}\right)
$$

With the previous $\tilde{M}$ remains,

$$
\tilde{M}=H G=\left(\begin{array}{cc}
-\frac{\gamma M^{*}}{\delta}+\frac{\beta N \delta\left(1-u_{1}\right)\left(1-u_{2}\right) T}{\delta c} & \frac{\beta\left(1-u_{1}\right) T}{c} \\
0 & 0
\end{array}\right) .
$$

Then, the the next-generation matrix evaluated in the equilibrium point in absence of infection $\bar{E}_{0}\left(\frac{\sigma}{\mu}, 0, \frac{\lambda}{\rho}, 0,0,0\right)$ yields as result:

$$
\tilde{M}=\left(\begin{array}{cc}
\frac{\sigma F}{\mu \delta c} & \frac{\sigma \beta\left(1-u_{1}\right)}{\mu c} \\
0 & 0
\end{array}\right)
$$
by:

Finally, the Eigenvalues of this matrix are $z_{1}=0$ and $z_{2}=\frac{\sigma F}{\mu \delta c}$, hence, the spectral radius is given

$$
R_{0}=p(\tilde{M}):=\operatorname{máx}\left(0, \frac{\sigma F}{\mu \delta c}\right)=\frac{\sigma F}{\mu \delta c}=\frac{\sigma N \beta\left(1-u_{1}\right)\left(1-u_{2}\right)}{\mu c} .
$$

which corresponds to the basic reproduction number.

Proof of Proposition 2. To study the number of positive roots of the equation (9), we keep in mind Descartes' Rule of signs, which establishes that the number of positive real roots of a polynomial equation with real coefficients equaled to zero is, if anything, equal to the number of sign changes produced between its coefficients [36]; hence, there would be no positive roots if $B$ and $C$ are at the same time positive. Thus, it will be proven that $B C<0$ as long as $R_{0}>1$ and $\alpha<\psi$, in effect, it is obtained that $R_{0}>1 \Longrightarrow c \mu \delta<\sigma F$

$$
\begin{aligned}
B & =-\gamma \lambda \psi F+\delta F \rho(\alpha-\psi)-\alpha \psi \sigma F+\alpha \psi \mu \delta c \\
& \leq-\gamma \lambda \psi F+\delta F \rho(\alpha-\psi)-\alpha \psi \sigma F+\alpha \psi \sigma F \\
& <0
\end{aligned}
$$

For the case $\alpha>\psi$ and $R_{0}>1$ we obtain $C<0$, in effect

$$
\begin{aligned}
C & =-\delta \rho^{2} F-c \mu \gamma \lambda \psi+c \mu \delta \rho(\alpha-\psi)-\sigma F \rho(\alpha-\psi) \\
& <-\delta \rho^{2} F-c \mu \gamma \lambda \psi+c \mu \delta \rho(\alpha-\psi)-c \mu \delta \rho(\alpha-\psi), \text { because } R_{0}>1 \Longrightarrow c \mu \delta<\sigma F \\
& =-\delta \rho^{2} F-c \mu \gamma \lambda \psi \\
& <0
\end{aligned}
$$

With which it is proven that if $R_{0}>1, B$ and then $C$ have contrary signs, that is, $B C<0$. 
Given that $B<0$ or $C<0$ two sign changes are produced in the equation (9), which is why according to Descartes' Rule of signs there will be a maximum of two positive roots. If there are two positive solutions, these are assumed as $\bar{T}_{1}^{*}$ and $\bar{T}_{2}^{*}$, where $\bar{T}_{1}^{*}<\bar{T}_{2}^{*}$. We then have:

1. There are two positive solutions, that is, two endemic equilibriums if $\bar{T}_{1}^{*}$ and $\bar{T}_{2}^{*}$ satisfy the conditions to obtain $\bar{M}^{*}>0$ (see (8)); that is:

$$
0<\overline{T_{1}^{*}}<\overline{T_{2}^{*}}<\frac{\rho}{\alpha} .
$$

2. There are no positive solutions, that is, there is no endemic equilibrium if $\overline{T_{1}^{*}}$ and $\overline{T_{2}^{*}}$ fulfill:

$$
\frac{\rho}{\alpha}<\overline{T_{1}^{*}}<\overline{T_{2}^{*}}
$$

3. There is a single endemic equilibrium point if $\overline{T_{1}^{*}}$ and $\overline{T_{2}^{*}}$ fulfill:

$$
0<\overline{T_{1}^{*}}<\frac{\rho}{\alpha}<\overline{T_{2}^{*}}
$$

To satisfy equations (A1) and (A2), it must be fulfilled that when evaluating in the cubic equation (9) the value of $\frac{\rho}{\alpha}$ is $>0$; however, by evaluating $\frac{\rho}{\alpha}$ in the equation (9) it remains:

$$
\begin{aligned}
& A\left(\frac{\rho}{\alpha}\right)^{3}+B\left(\frac{\rho}{\alpha}\right)^{2}+C\left(\frac{\rho}{\alpha}\right)+D=0 \\
& \Leftrightarrow \delta \psi F \frac{\rho^{3}}{\alpha^{2}}+\delta F(\alpha-\psi) \frac{\rho^{3}}{\alpha^{2}}-\gamma \lambda \psi F \frac{\rho^{2}}{\alpha^{2}}+\psi(\delta c \mu-\sigma F) \frac{\rho^{2}}{\alpha}-\delta F \frac{\rho^{3}}{\alpha}=0 \\
& \Leftrightarrow-c \mu \gamma \lambda \psi \frac{\rho}{\alpha}+\delta c \mu(\alpha-\psi) \frac{\rho^{2}}{\alpha}+F \sigma(\psi-\alpha) \frac{\rho^{2}}{\alpha}+\sigma F \rho^{2}-\delta c \mu \rho^{2}=0 \\
& \Leftrightarrow-\gamma \lambda \psi F \frac{\rho^{2}}{\alpha^{2}}-c \mu \gamma \lambda \psi \frac{\rho}{\alpha}<0 .
\end{aligned}
$$

Hence, the equation is fulfilled (.3) and it is concluded that if $R_{0}>1$ a single point of equilibrium exists in presence of virus in the system, which is $\overline{E_{1}}=\left(\bar{T}, \bar{T}^{*}, \bar{M}, \bar{M}^{*}, \bar{V}, \bar{W}\right)$ given by (8).

Proof of Proposition 3. To study the local stability, linearization of the system is performed, that is, the linear approximation will be found of the functions of the system of equations in the equilibrium points $\overline{E_{0}}$ and $\overline{E_{1}}$. Thus, the Jacobian matrix is evaluated in the trivial equilibrium point $\overline{E_{0}}=\left(\frac{\sigma}{\mu}, 0, \frac{\lambda}{\rho}, 0,0,0\right)$.

Whose characteristic polynomial is given by:

$$
p(z)=(-\mu-z)(-c-z)(\rho+z) Q(z)
$$

where,

$$
Q(z)=z^{3}+(\rho+\delta+c) z^{2}+\left(\delta \rho+c \rho+\delta c-\frac{\sigma}{\mu} F\right) z+\left(\delta c \rho-\frac{\sigma}{\mu} \rho F\right) .
$$

Finally, it is equaled to zero $p(z)$ to obtain the Eigenvalues and conclude about the system's stability through the linearization theorem. Thus, obtaining the following three negative values of $z$, $z=-\mu ; z=-c ; z=-\rho$ and a cubic equation

$$
Q(z)=\frac{1}{\delta c} z^{3}+\frac{\rho+\delta+c}{\delta c} z^{2}+\left(\frac{\rho}{c}+\frac{\rho}{\delta}+1-R_{0}\right) z+\rho\left(1-R_{0}\right)=0
$$

If $R_{0}<1$ then (.4) does not present sign changes in its coefficients with which, due to Descartes' rule, it is concluded that the roots of the cubic equation are all negative. Thus, through the linearization theorem, it is stated that $\bar{E}_{0}$ is locally asymptotically stable (LAE). If $R_{0}>1$, the coefficient 
$\rho\left(1-R_{0}\right)<0$ and the coefficient $\left(\frac{\rho}{c}+\frac{\rho}{\delta}+1-R_{0}\right)$ could be positive or negative, in any of both cases the equation will only have a change of sign, which is why it is concluded, through Descartes's rule of signs that there will be a positive real root and, hence, it is established that if $R_{0}>1$, the trivial equilibrium point $\overline{E_{0}}$ is unstable.

Now, upon evaluating the Jacobian matrix on the point of stability $\bar{E}_{1}$, it is found that the characteristic polynomial is in the form:

$$
p(z)=A_{0} z^{6}+A_{1} z^{5}+A_{2} z^{4}+A_{3} z^{3}+A_{4} z^{2}+A_{5} z+A_{6}
$$

Given that algebraic difficulties arise when trying to find Eigenvalues, it is established as a conjecture that equilibrium $\overline{E_{1}}=\left(\bar{T}, \overline{T^{*}}, \bar{M}, \overline{M^{*}}, \bar{V}, \bar{W}\right)$ is LAE when $R_{0}>1$, which will be illustrated numerically.

Sample Availability: Samples of the compounds ...... are available from the authors. 